\title{
The Dynamic Effects of Bundling as a Product Strategy
}

\author{
Timothy Derdenger \& Vineet Kumar*
}

\begin{abstract}
Several key questions in bundling have not been empirically examined: Is mixed bundling more effective than pure bundling or pure components? Does correlation in consumer valuations make bundling more or less effective? Does bundling serve as a complement or substitute to network effects? To address these questions, we develop a consumer-choice model from micro-foundations to capture the essentials of our setting, the handheld video game market. We provide a framework to understand the dynamic, long-term impacts of bundling on demand. The primary well known explanation for the profitability of bundling relies on homogenization of consumer valuations. However, we find bundling can be effective through a previously unexamined mechanism of dynamic consumer segmentation that can operate even when homogenization might not be effective. In the absence of bundling, we find that sales of both hardware and software components decrease, and consumers who had previously purchased bundles might delay purchases, resulting in lower revenues. We also find that mixed bundling dominates pure bundling and pure components in terms of both hardware and software revenues. Investigating the link between bundling and indirect network effects, we find that they act as substitute strategies, with a lower relative effectiveness for bundling when network effects are stronger.
\end{abstract}

${ }^{*}$ Both authors contributed equally to this paper, and are listed in alphabetical order.

Tim Derdenger is Assistant Professor in Marketing \& Strategy, Tepper School of Business, Carnegie Mellon University. e-mailderdenge@andrew.cmu.edu.

Vineet Kumar is Assistant Professor of Business Administration, Marketing Unit, Harvard Business School, Harvard University. e-mail|vkumar@hbs.edu.

The authors would like to thank Sunil Gupta, Brett Gordon and Minjung Park and for comments on an earlier draft of the paper.

They gratefully acknowledge feedback from seminar participants at Yale University, Catholic University of Leuven, and the University

of Zurich as well as conference participants at Marketing Science, Northeastern Marketing Conference and the UT Dallas FORMS conference. All errors remain their own. 


\section{Introduction}

Bundling, the practice of including two or more products within a separate product bundle, is arguably the most flexible element of product strategy, since the component products are already available. Bundling is commonly used in a diverse range of industries, with examples including fast food (value meals at McDonalds), insurance (automobile, home and umbrella), telecommunications (home internet \& phone service). Bundling is especially common in technology and content industries, ranging from music albums (bundle of songs), newspapers (bundle of articles) to cable television (bundle of channels). Bundling could involve both similar products (e.g. season tickets), and dissimilar or complementary products (e.g. consoles and video games).

It is interesting to note that record companies make both singles and entire albums available for purchase, whereas most newspapers or online news sites commonly do not allow purchase of individual articles. We thus find two types of bundling commonly used in practice: pure bundling refers to the practice of selling two or more discrete products only as part of a bundle, whereas mixed bundling refers to the practice of selling a bundle of the products as well as the individual products themselves 1 Another example of this dichotomy occurs in office productivity suites: Microsoft only sells Microsoft Word as part of Microsoft Office (pure bundling), whereas Apple has moved away from marketing the corresponding Pages software as part of the iWork bundle, and it is currently available as a pure product. In the smartphone market, both Apple and Google bundle software applications like Maps and GPS with the hardware and operating system as a pure bundle. The variety of bundling possibilities in each market and its ease of implementation make bundling an important product strategy decision that hold significant potential for the firm.

Our objective is to empirically examine the effectiveness of bundling as a product strategy, especially to understand the dynamic effects of bundling in markets with complementary products, where consumers could derive additional utility from having both products, e.g. hardware and software, as opposed to having just one or the other [Nalebuff, 2004]. We seek to understand and answer the following research questions:

1. Cannibalization and Market Expansion: Does bundling result in cannibalization of pure component products or does it increase overall sales of both products?

2. Bundling Types: Are bundles equivalent to the product components purchased together? Is mixed bundling (both bundle and component products are available) more effective than pure components or pure bundling?

3. Complementarity and Network Effects: Does the presence and strength of network effects or complementarity make bundling relatively more or less effective?

\footnotetext{
${ }^{1}$ Note that pure components refers to the strategy of selling individual products without bundling.
} 
We develop a model to study these dynamics in the setting of handheld video game consoles (hardware) and games (software), where consumers purchase products of a durable nature, with intertemporal tradeoffs playing a key role in decision making.

Much prior research has focused on how bundling results in the homogenization of consumer valuations Adams and Yellen, 1976, Schmalensee, 1984a, McAfee et al., 1989. The central idea is that a monopolist can use bundles to reduce consumer heterogeneity and create a more homogeneous distribution of consumer valuations, leading to better extraction of consumer surplus. In the limit, it is easy to see that a monopolist facing a market of identical consumers can use uniform pricing to achieve complete extraction of consumer surplus.

Our primary contributions are along two dimensions. First, we uncover an additional indepedent mechanism for bundling in dynamic settings, based on the notion of the bundle serving as a product to achieve more effective dynamic consumer segmentation. We find that bundles serve a role similar to an additional product in the firm's product offering, and that the presence of bundles causes certain segments of consumers to advance their purchases from later periods to earlier periods, resulting in more effective consumer segmentation over time. We also find that the presence of bundles increases the sales of both component products, thus magnifying its beneficial effects. Broadly, we find that bundling can be effective under a much wider range of conditions in a dynamic setting than proposed by the literature. Our findings provide more insight into an alternative mechanism that can make bundling especially effective in markets with intertemporal tradeoffs and significant consumer heterogeneity, e.g. durable goods like automobiles, consumer electronics and in technology markets where tradeoffs on when to purchase as especially important.

Since prior research on bundling has been mostly been developed for static settings, the proposed mechanism is a novel discovery. The dynamic consumer segmentation is indeed most effective when consumer valuations for the two product components is positively correlated, i.e. consumers have high valuations for both hardware and software, or low valuations for both. In such a market, we find that consumers with low valuation for hardware and software intertemporally substitute and accelerate their hardware purchases when bundles discounted from sum of component prices are available as an option. As we might expect, we find that heterogeneity plays a crucial role in how bundling becomes effective, with more heterogenity increasing the effectiveness of bundling. The company's revenues for hardware are increased with bundling, and the intertemporal substitution effect of the low segment consumers segment plays a significant role. Thus, we find that bundling increases revenues because consumers with low hardware valuations accelerate their purchases in the presence of bundles, but high valuation consumers still find individual consoles to provide flexibility in making software purchases, and of high enough value not to substitute away from a choice of pure console.

Second, we empirically examine the nature and effectivenenss of different approaches to bundling, i.e. mixed bundling versus pure bundling; the theoretical literature has found support for either choice to be dominant depend- 
ing on the setting and conditions. We find that relative to pure components, mixed bundling enhances revenues for both hardware and software, whereas pure bundling diminishes sales of both types of products. Bundles are separate products in their own right, amd are not equivalent to the sum of component products, and they serve a distinct role the firm's product offering. Finally, we examine the interaction between bundling and network effects, and find that they serve as substitutes, i.e. bundling is more effective in settings with weaker network effects, suggesting that managers might find it useful to bundle in settings and time periods when the network effect is weaker.

From a methodological perspective, we provide a new strategy to identify correlation between consumer preferences for complementary products, i.e. hardware and software, using aggregate data. Our identification argument is based on how the tying ratio (ratio of software sales to hardware installed base) varies dynamically and does not rely on the presence of bundles in the consumer's choice set. We also incorporate an explicit microfoundations-based link between the hardware and software markets (indirect network effect), so that consumers place a higher value on hardware when there are more and better games available at lower prices in the software market, and when more games are expected to become available in future periods. Most other research in the literature model the indirect network effect with a reduced form approach, often using the number of products available as proxy, e.g. Dube et al., 2010, while expanding on other dimensions.

Our model incorporates the durable nature of products, so that consumers choose between purchasing versus waiting. The timing of the model is as follows: consumers who do not own hardware must decide whether or not to purchase a console or bundle each period until they make a purchase. When consumers purchase a console or bundle, they exit the hardware market and enter the software market. In each period in the software market, consumers make decisions regarding whether and which game to purchase, depending on the available choice set for games.

The framework uses the approach of tractably characterizing an inclusive value, and builds upon dynamic demand frameworks Melnikov 2001, Gowrisankaran and Rysman 2013, which in turn are based on the workhorse BLP model [Berry et al. 1995], and we extend this framework along a number of dimensions. We approximate the expected future value of both consoles and games separately as the inclusive value for hardware and for software. The inclusive value is the present discounted value of making a purchase in the current period, and consumers form expectations over the evolution of the inclusive value. The inclusive value abstraction is designed to tractably capture the possible variations in product availability, pricing and other unobservable factors that might evolve over time, collapsing multiple dimensions of the state space to two dimensions, one each for the hardware and software markets. Similar to other dynamic demand models, we also abstract away supply-side decisions like product development and design, although we do evaluate different supply-side configurations for bundling as counterfactuals. 
Although we use the setting of handheld video game consoles and games, the mechanisms we propose are more general and could be found in other dynamic settings. Our findings point to practically relevant and highly significant results for product strategy and management. Since bundles are created rather easily in most contexts, we expect this to be a practical and easily achievable option for firms across a variety of industries.

\section{Related Literature}

The phenomenon of bundling, both of the pure and mixed varieties has received much attention in the theoretical literature in marketing and economics. However, there has been little empirical understanding of the effects of bundling, which is clearly required to characterize both the short-term product substitution effects as well as dynamic long-term demand enhancing effects we seek to study.

A survey of the major practical tradeoffs in constructing bundles at a conceptual and theoretical level is presented in Venkatesh et al. [2009]. Bundling has traditionally been considered a price discrimination strategy to extract more surplus from consumers who have heterogeneous valuations for different products, as illustrated in early work by Adams and Yellen 1976, and modeled in detail by Schmalensee 1984a]. These papers recognize that consumer heterogeneity is the primary reason why a monopolist would not be able to extract full surplus from consumers, and contribute the key idea that heterogeneity in valuation across consumers can be diminished by bundling multiple products. Recall that consumer heterogeneity is a primary reason that a monopolist cannot fully extract all surplus from consumers. The reduction in heterogeneity due to bundling happens because the variance in the sum of product valuations is lower than the sum of variances in product valuations, which then allows a monopolist to more effectively extract surplus.

Recent research on mixed bundling indicates that this strategy is likely to be more profitable when the products to be bundled are sufficiently asymmetric in production costs as well as network effects [Prasad et al., 2010, whereas more similarity between products makes pure bundling or pure components profitable. It is noteworthy that the authors point to the lack of empirical research at the confluence of network effects and bundling, echoing more general calls for an empirical measurement of the market effects of bundling [Kobayashi, 2005].

A contrary view advancing the optimality of pure bundling in the context of a monopolist with products of different qualities indicates that pure bundling prevails over pure components (i.e. individual products), when consumer valuations for the products are super-additive. Given the sheer number and variety of results that apply under different conditions, the lack of empirical study of mixed bundling is especially striking.

Our focus on dynamics and complementary goods in a two-sided market as opposed to examining related goods of the same type makes both our methodology and result very different from other empirical work on bundling related to content industries like music and cable TV, where studies have demonstrated that mixed bundling can actually reduce revenues and profitability compared to pure bundling [Crawford, 2008, Elberse, 2010; in contrast, 
we find that dynamics make bundling more profitable.

A related literature on tying, where a product is only offered for purchase in conjunction with another product has received significant attention for its anti-competitive effects. Tying can be best thought of as an extreme form of bundling, involving a primary good and an aftermarket good, where the consumer is essentially forced to purchase the aftermarket product, unless she bears switching costs in switching to a different primary good. Tying can be used as a coordination tool in platform settings, and can actually help raise social surplus Amelio and Jullien. 2007, and can also have channel implications resulting from consumer choice of retailers Hartmann and Nair. 2010 .

\section{Industry Structure and Data Description}

We focus on the handheld videogame market, studying its structure during the years 2001-2005. During these years, the industry resembled a monopoly market, with Nintendo as the dominant company. The setting is essentially a platform market, with consumers purchasing hardware consoles and software titles, and with a monopolist providing the hardware and some software, and independent third-party producers creating additional software titles compatible with the hardware $2^{2}$

In platform markets like this one, consumers typically purchase the hardware first before they enter the market for software titles. However, consumers do consider the number and quality of software titles that exist for the platform when they make their purchase decision, as well as how the number and quality of titles is expected to evolve in the future, consistent with the literature on indirect network effects Katz and Shapiro, 1994.

We distinguish between integrated developers such as Sony in the high definition DVD market or Nintendo in the video game industry, and independent software developers. Integrated content is produced by the platform's own content design studio, whereas independent software is produced by firms not controlled by the platform manufacturer. In addition to selling access to consumers and producing content, the platform can also offer a bundle of the hardware and software developed by its integrated development studio, as is the case in our setting. Both hardware and software, including consoles, games and bundles were primarily marketed and sold through brick-and-mortar retailers in the time period corresponding to our data. The bundles were packaged by the manufacturer (Nintendo), and only included select games from their own integrated studio.

\section{Data}

The data used in this study originates from the marketing group NPD; they track sales and pricing for the

\footnotetext{
${ }^{2}$ See Kaiser (2002), Caillaud and Julien 2003, Rochet and Tirole 2006, Rysman 2004, Kaiser and Wright 2006, Armstrong and Vickers 2010, Hagiu 2006 for general literature on two-sided platform markets.
} 
video game industry and collect data using point-of-sale scanners linked to a majority of the consumer electronics retail stores in the United States. NPD extrapolates the data to project sales for the entire country. Included in the data are quantity sold and total revenue for two consoles and three bundles and all of their compatible video games, numbering approximately 700 .

The data set covers 45 months starting in June 2001 and continues through February 2005, during which time Nintendo was a monopolist in the portable video game market, and before Sony's PlayStation Portable entered the market. Nintendo was a multi-product monopolist producing two versions of its very popular Game Boy Advance (GBA) console as well as a portfolio of games to be played on its console. Each version was internally identical, but the second version dubbed the GBA SP was reoriented with the display lying horizontally rather than vertically. The GBA SP looked like a mini laptop computer and was close to half the size of the original GBA. Moreover, it is usually the case with the introduction of a new device that new games are released which are not backwards compatible. However, with the introduction of the GBA SP, the internal hardware of both devices were identical, and both devices could share the same set of games.

The target market of these two devices was younger kids rather than teenagers or young adults, which were the targeted demographic segments for the home video game console. Portable or handheld consoles differ from traditional home video game consoles, since they are mobile, with the size of the device being no larger than an adult hand. The devices are designed to easily travel with a consumer and can be played in a car or airplane, whereas a home console is restricted to locations with a television display and electricity.

General statistics of the portable video game industry are provided in the tables below. We also present a plot of aggregate sales data for hardware and software in Figure $23^{3}$ In Tables 1 and 2 we present statistics on the release date, total units sold and the number of months on the console market, average (min and max) prices and total units sold for each of the two standalone consoles and three bundles.

Table 1: Portable Console Market Statistics

\begin{tabular}{llll}
\hline & Release Date & Units & Months on Console Market \\
\hline Nintendo & & & \\
GBA & June 2001 & $12,821,233$ & 45 \\
GBA SP & March 2003 & $13,070,720$ & 24 \\
GBA w / Mario Kart & November 2001 & 215,394 & 29 \\
GBA w / Mario Advance 2 & November 2002 & 199,225 & 17 \\
GBA SP w/ Mario Advance 4 & November 2003 & 149,065 & 4 \\
\hline
\end{tabular}

\footnotetext{
${ }^{3}$ Sales data is presented in its raw and deseasoned form, where the data is deseasoned with the use of the X11 program from the US Census.
} 
Table 2: Portable Console and Bundle Prices

\begin{tabular}{lllll}
\hline & Average Price & Max Price & Min Price & Independent Games Sold \\
\hline Nintendo & & & & \\
GBA & $\$ 72.00$ & $\$ 94.46$ & $\$ 52.37$ & \\
GBA SP & $\$ 93.73$ & $\$ 100.30$ & $\$ 70.60$ & \\
GBA w / Mario Kart & $\$ 86.17$ & $\$ 150.54$ & $\$ 61.50$ & $2,027,636$ \\
GBA w / Mario Advance 2 & $\$ 67.33$ & $\$ 71.73$ & $\$ 56.60$ & $2,438,732$ \\
GBA SP w/ Mario Advance 4 & $\$ 97.62$ & $\$ 99.85$ & $\$ 94.92$ & $1,673,304$ \\
\hline
\end{tabular}

Figure 1: Sales for Hardware and Software

(a) Monthly Hardware Sales

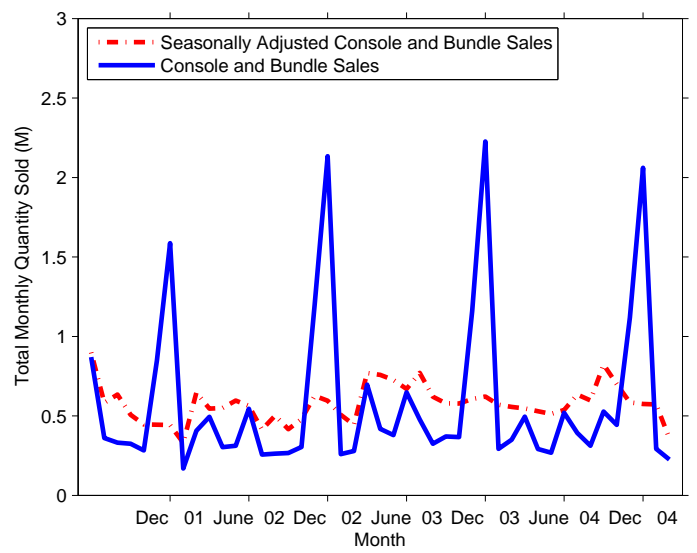

(b) Monthly Software Sales

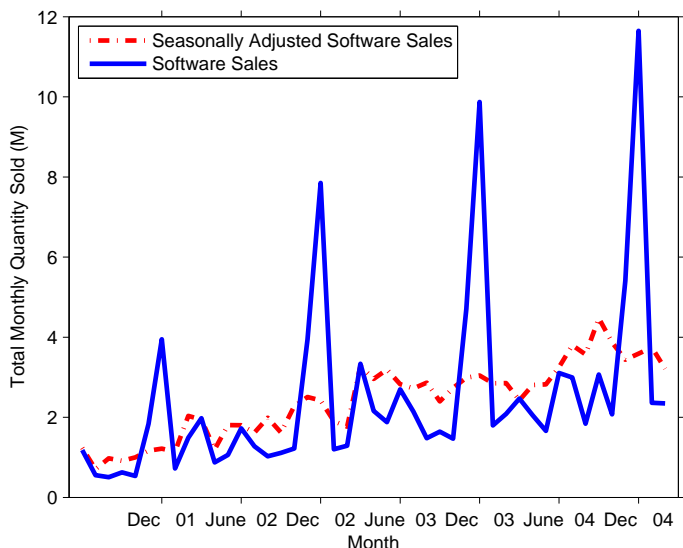

Figure 2: Hardware Market Sales and Prices

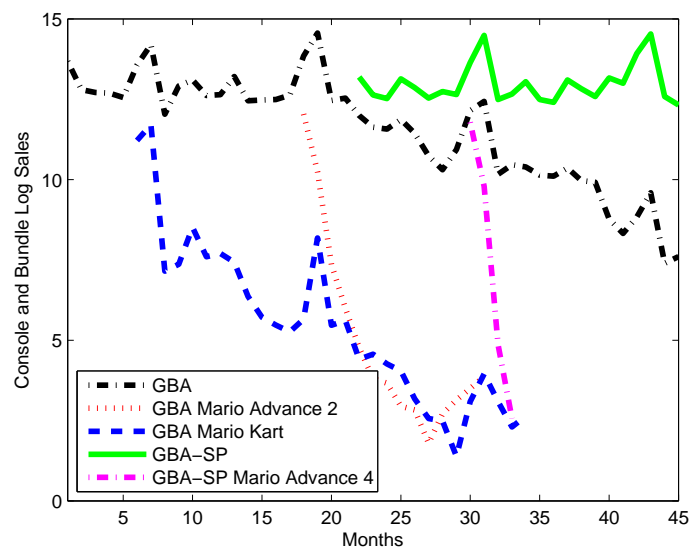

(a) Hardware Console and Bundle Sales

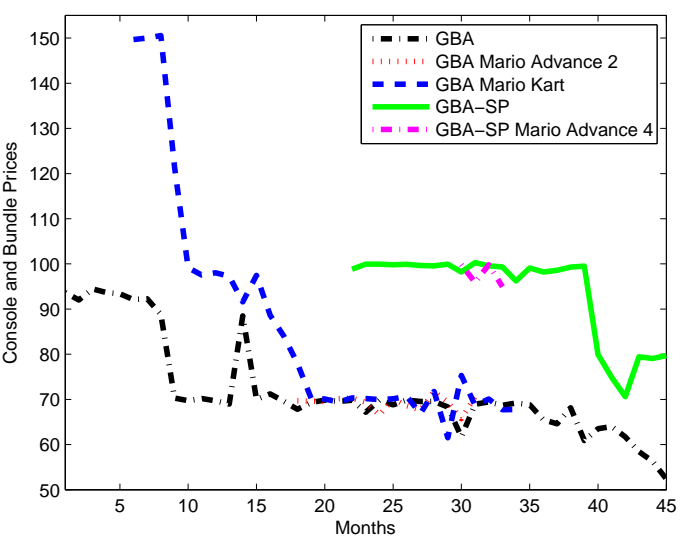

(b) Hardware Console and Bundle Prices

We note several interesting observations from figures 1 and 2 , which illustrate the sales of consoles and bundles over time. First, we see that there are significant dynamics in this marketplace. Both sales and prices of products are generally declining over the period of 45 months in the data, but there are also periods of stability and even 
Table 3: Dynamic Software Sales Regression

\begin{tabular}{lcc} 
Units sold in period $t$ is Dependent Variab \\
\hline Units & Coefficient & Std. Error \\
\hline Units $_{t-1}$ & 0.2739 & 0.006 \\
I(bundle $)_{t}$ & 14628.06 & 3196.003 \\
I(bundle $)_{t-1}$ & 15768.94 & 2174.108 \\
price $^{\text {age }}$ & -1169.34 & 51.094 \\
age & -872.04 & 287.956 \\
age $^{2}$ & 39.22 & 1.6091 \\
\hline \hline
\end{tabular}

Note: Month fixed effects included

increases. Second, we observe that bundles can be both short-lived, like the "Gameboy Advance SP with Mario Adv 4" which is only available for 4 periods, or can persist over a long period of time, like in the case of "Gameboy Advance with MarioKart." Third, we observe sales for both GBA and GBA SP after the GBA SP is introduced approximately in the middle of the time spanned by our data. The video game industry exhibits a large degree of seasonality, with new products typically being introduced during the holiday season. Sales of both old and new consoles typically also see significant increases in the months of November and December.

Nintendo also released three bundles over time - the first being a GBA device bundled with the hit game Mario Kart in November 2001. Additionally, all bundled games were high quality hit video games each selling over one and half million standalone units. These data demonstrate the importance of including the presence of bundles, and accounting for the large degree of seasonality as well as dynamic changes in consumer choice sets in our modeling framework.

To get an approximate idea of the dynamics of video game software sales (in units), we regress the current period sales as a function of lagged sales, current price, age and age $^{2}$ as well as whether there is currently a bundle present or was present in the previous period:

$$
s_{g, t}=\theta_{1} s_{g, t-1}+\theta_{2} \mathbf{I}\left(\text { bundle }_{t}\right)+\theta_{3} \mathbf{I}\left(\text { bundle }_{t-1}\right)+\theta_{4} p_{g, t}+\theta_{5} \text { age }+\theta_{6} a g e^{2}+\omega_{g, t}
$$

where $\omega_{g, t}$ is distributed iid as a standard normal random variable. We estimate the above specification using the Arellano-Bond GMM estimation procedure given the endogeneity of the one period lagged measure of the dependent variable and price. Standard instruments of lagged regessors are used.

Examining the results of the regression in Table 3, we find that having a bundle sold in period $t$ or $t-1$ is associated with increased software sales, age appears to have a negative effect on sales, while the positive coefficient on $a g e^{2}$ indicates that the magnitude of the marginal effect is diminished as the game ages. This finding implies that there is likely to be pent up demand for games, and significant sales are achieved quickly after product release, beyond which sales decline. Note that the above analysis is not intended to serve as a causal account, given the multiple concerns it might raise, including endogeneity. Rather we use these results along with the model-free evidence in Figure 1 to motivate the need for investigating the dynamics of the market by modeling the micro- 
Figure 3: Model Timeline

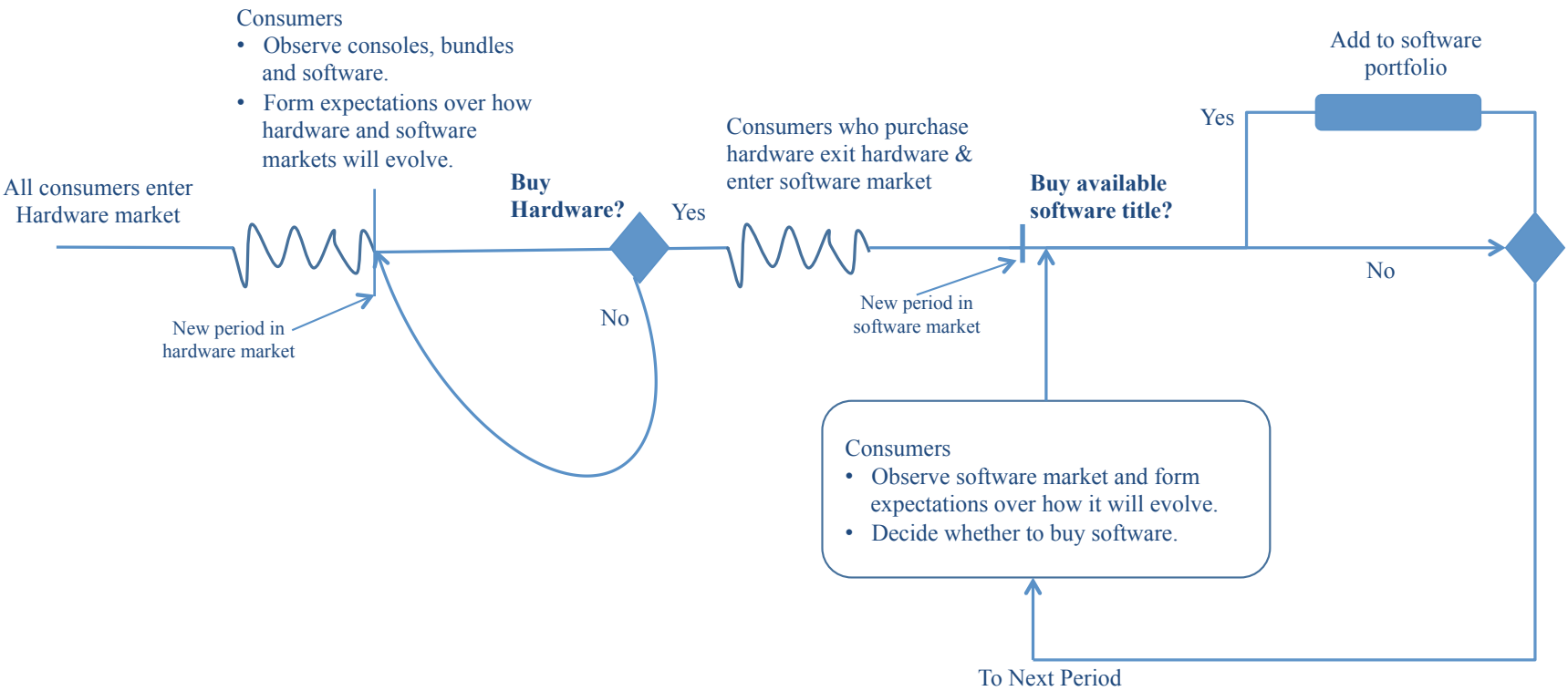

foundations of consumer decisions, which help in explaining and understanding these dynamic data patterns.

\section{Model}

We develop a model that captures the essentials of our institutional setting and consumer buying behavior, and is suitable for use with aggregate market data. We consider first the trade-offs faced by consumers who already own a console, and are in the market for video games, and then proceed to examine how consumers make decisions in the console market. We now outline the timing of the consumer decision journey, which begins in the hardware market. When a consumer is present in the hardware market, she makes a discrete choice from the set of available consoles or bundles or decides not to make a purchase. After purchasing a hardware unit, she exits the hardware market and enters the software market, where she may purchase a video game in each period, or make no purchase. The timeline of the consumer journey is detailed in Figure 3.

There are several specific details of our institutional setting that must be captured by our model to ensure that the effects of bundling are appropriately characterized:

- Dynamics: There are several sources of dynamics in the model, including firm driven variations such as price changes, variation in product availability for consoles, video games and bundles, as well as consumer dynamics due to entry and exit of consumers from the hardware and software markets. In durable goods settings such as ours, consumers face the option of delaying buying, and their purchases continue to provide flow utility in future periods following purchase. Another issue that contributes to dynamics in our setting is the inherent seasonality in purchases of videogame consoles and games, which we aim to explicitly incorporate.

- Interconnection between Hardware and Software Markets: The setting involves sequential and in- 
terconnected decisions by consumers. Consumer typically purchase hardware first, followed by a stream of software purchases over time. Such a sequenced purchase pattern implies that a consumer's current choice can alter the future choice set. Consumers account for the utility from having access to a stream of software products to use in conjunction with their hardware when determining whether or not to purchase hardware. We cannot abstract away from the software market since we would not be able to construct an appropriate model for a bundle in that case.

- Heterogeneity: Consumers are typically heterogeneous in valuation for both hardware and software, and this distinction becomes critical to capture accurately, since the consumer mix varies over time as they make purchase decisions and enter or exit markets. For example, consumers who value hardware more could purchase early, leading to the population of consumers remaining in the hardware market exhibiting a diminishing value for hardware over time. A second critical factor in the bundling context is correlation between valuation for hardware and software, which is dependent on consumer heterogeneity and is expected to have a significant impact on the effectiveness of bundling. With no heterogeneity across consumers, note that we have zero correlation.

There are several models of demand that allow for individual consumer choices in a differentiated marketplace to be aggregated up to the market-level demand, which can be estimated when using sales data. Most structural approaches are based on Berry et al. 1995] (BLP, hereafter), who demonstrated how to incorporate unobservable heterogeneity among individual consumers in a static framework where consumers choose between a set of discrete alternatives.

The BLP framework has been extended to incorporate dynamic effects with forward-looking consumers $\mathrm{Mel}_{-}$ nikov, 2001, Schiraldi, 2011, Carranza, 2010, Gowrisankaran and Rysman, 2013. We base our model on the approach suggested by Melnikov 2001 and further extended by Gowrisankaran and Rysman 2013 (G\&R, hereafter), who formulate a model of dynamic demand where the evolution of the market is captured by a single inclusive value variable representing dynamic purchase utility that is both specific to an individual consumer and varying over time. This specification has an intuitive interpretation and captures the dynamics in a parsimonious manner, enabling the development of a tractable model that aggregates the behavior of forward-looking consumers, and allows for estimation with market-level data. The idea of collapsing the entire state space into an inclusive variable allows us to capture multiple sources of dynamics in a tractable manner. Such dynamic changes might include a rich array of possibly uncertain dimensions, e.g. the introduction of new hardware and software products and their features, price changes, promotions that are unobservable to the researcher. An alternative way to approach this problem is to choose a small number of primary dimensions of interest, and model a consumer expectation process for those specific variables, e.g. Gordon [2009], in his study of the personal computer market considers the CPU Speed as the primary quality dimension, and the price as an additional dimension which helps keep the state space tractable. 
To aid in exposition, we begin our description of the consumer utility in reverse chronological order, beginning with software purchase decisions made by consumers. We must emphasize that, like in other durable goods settings, consumers examine the dynamic stream of utilities obtained from a purchase when they make the decision to purchase or not purchase, and the utilities in $\S 3.3$ and 3.3 below are not sufficient for consumer decision-making, which is detailed in $\S 3.3$.

\subsection{Consumer Model for Software (Video Games)}

We consider here consumers who own a hardware unit (console or bundle) and are in the market for software (video games). The potential market for video games in a period is thus driven by the installed base, or number of consumers who have purchased hardware prior to that period 4 Consumers face a choice of which video game to purchase, and we denote the choice set of video games (or software) available for purchase in period $t$ as $\mathbf{S}_{\mathbf{t}}$, which includes the "no purchase" option, 0. Each software title or video game is assumed to compete with other software titles, which allows consumers to substitute across games in each period, and across time periods.

We model a consumer as purchasing at most one video game in each period. We begin with a purchase period utility (or just "period utility") that represents the utility obtained during the period of purchase. For consumer $i$, her period utility from purchasing game $g$ in period $t$ is:

$$
\begin{aligned}
u_{i g t}^{s} & =\frac{1}{\psi}\left(\tilde{\alpha}_{i}^{s}+\tilde{\alpha}^{w, s} w_{g, t}^{s}+\tilde{\chi}_{g t}+\tilde{\alpha}^{p, s} p_{g, t}+\eta_{\tau(t)}^{s}\right)+\epsilon_{i g t} \\
& =\underbrace{\overbrace{\alpha_{i}^{s}}^{\text {Software Effect }}+\overbrace{\alpha^{w, s} w_{g, t}}^{\text {Observable Characteristics }}+\overbrace{\chi_{g t}}^{\text {Unobservable Characteristics }}}_{\text {Software Flow Utility: } v_{i g t}^{s}}+\alpha^{p, s} p_{g, t}+\underbrace{\eta_{\tau(t)}^{s}}_{\text {Seasonal Effect }}+\epsilon_{i g t}^{s}
\end{aligned}
$$

In the above consumer utility expression, $w_{g, t}$ represents the observable characteristics of the game $g$ in period $t$ including variables like age, genre etc. of the game. The unobservable game-period effect is represented by $\chi_{g, t}$, and rationalizes market sales over time periods, as is typical in BLP-type models. The price of the software game $g$ in period $t$ is denoted by the variable $p_{g, t}$. We capture seasonality using the month fixed effect term $\eta_{\tau(t)}^{s}$, where $\tau(t)=t \bmod 12$ represents the month of the year. The term $\epsilon_{i, g, t}^{s}$ is comprised of idiosyncratic Type I extreme value shocks independent across consumers, games and time periods.

The coefficient $\alpha_{i}^{s}$ represents the value that individual $i$ attaches to owning any software game, whereas $\alpha^{w, s}$ represents consumer valuation for software characteristics and $\alpha^{p, s}$ denotes the price coefficient for software. The parameter $\psi$ is a scaling parameter, and allows us to compare the utilities of hardware and software, and is the utility normalization factor, since we set the error terms for both hardware and software to have the same variance

\footnotetext{
${ }^{4}$ In our setting with a monopolist console manufacturer, all games are compatible with each console available on the market, so there are no additional compatibility variables to be tracked for each game.
} 
$\left(\frac{\pi^{2}}{6}\right.$ for a Type-I extreme value random variable). An alternative way to interpret $\psi$ is based on the degree to which the consumer utility is based on idiosyncratic shocks factors for software relative to hardware.

Consumers continue to obtain flow utility in periods following purchase. The terms denoted by $v_{i g t}^{s}$ in the utility are persistent software flow utility when consumer purchases game $g$ in period $t$, since software is durable. The flow utility is persistent across time periods, whereas the other terms in the period utility are only obtained during the period when the consumer makes a purchase. Consumer $i$ thus receives period utility $u_{i g t}^{s}$ in the period of purchase $t$, and continues to receive flow utility $\left(v_{i g t}^{s}\right)$ in all periods $\omega>t$ after purchasing, where the flow utility is fixed during the period of purchase.

Since consumers are forward looking and the product is a durable good, consumers do not make the decision to purchase based only on the above period utility or flow utility - these serve as "building blocks" for the consumer decision making process, detailed in 3.3 . The consumer has expectations about the choices she might make in the future and how her current choice would impact the future, she would continue obtaining flow utility in periods following a purchase.

\subsection{Consumer Model for Hardware (Consoles and Bundles)}

We develop a model of hardware choice, where each consumer considers whether or not to purchase hardware from the set of available consoles $\mathbf{J}_{\mathbf{t}}$ or bundles $\mathbf{B}_{\mathbf{t}}$. The overall choice set for a consumer in the hardware market is then $\mathbf{H}_{\mathbf{t}}=\{\mathbf{0}\} \cup \mathbf{J}_{\mathbf{t}} \cup \mathbf{B}_{\mathbf{t}}$. We model only consumers who have not purchased a console or a bundle to make up the hardware market of potential buyers for bundles. Although consumers consider the entire set of hardware available when making a purchase decision, for simplicity of exposition we first outline the utility specification for consoles, followed by bundles. Consumers who make a hardware purchase exit the hardware market permanently. Note that choice sets are allowed to vary over time.

\section{Consoles}

Consider the decision process when only consoles are available in the market: in each period, consumers can choose to purchase a console, provided they have not already purchased a console in the past $5^{5}$ Consumer $i \in \mathbf{I}$ determines in period $t \in \mathbf{T}$ whether or not to purchase console $j \in \mathbf{J}_{\mathbf{t}}$, and we denote this decision as $d_{i j t} \in\{0,1\}$. Consoles are durable and consumers receive a stream of flow utilities in all periods following a purchase. If consumer $i$ decides

\footnotetext{
${ }^{5}$ For households with multiple users that purchase multiple consoles, our model would treat them as separate consumers, in line with the current literature on aggregate demand models.
} 
to purchase console $j$ in period $t\left(d_{i j t}=1\right)$, he will obtain a purchase period utility given by 6

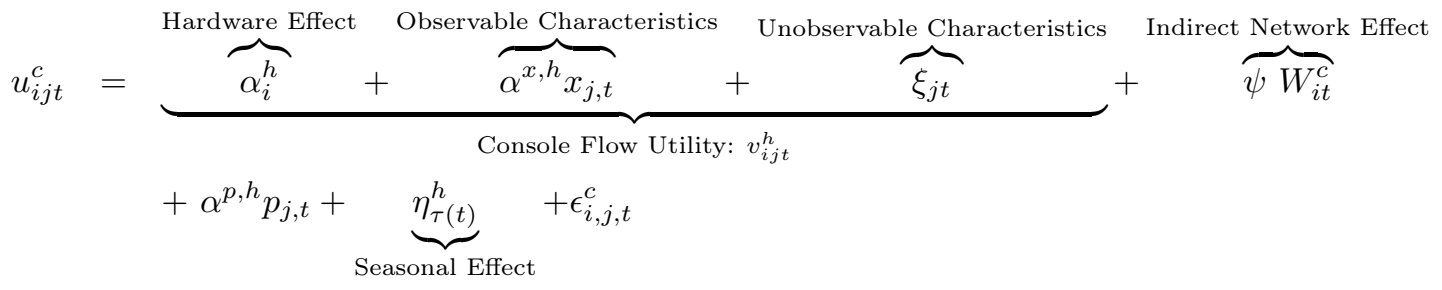

Each console is characterized by both observable product characteristics $x_{j, t}$ and an unobservable characteristic $\xi_{j t}$, which may vary both over time as well as across consoles. Note that the unobserved characteristic $\xi_{j t}$ is observed by consumers and accounted for by the console manufacturer, but is not observed by the researcher. Consistent with BLP, this characteristic could include product characteristics like style and design and usability as well as all other factors that are not present in the data.

The period utility of a console is also directly related to the games available for the consumer to purchase, and should incorporate the consumer's expectation of how the software market might evolve after purchasing hardware. We thus include the utility from software into the consumer's hardware utility function, using the term $W_{i t}^{c}$ to represent the present discounted scaled software utility available for the platform in period $t$ and the consumer's expectation of how it may evolve in the future. There are two ways to conceptualize the indirect network effect. The issue here is whether the expected value of software purchases is obtained as a flow utility after the consumer has purchased hardware and entered the software market. We might consider two viewpoints on this matter. First, if we interpret $W_{i t}^{c}$ as the option value of being present on the software market, then we would expect the consumer to obtain it as part of the flow utility. On the other hand, if it's interpreted as purely the expected discounted flow of utilities from optimal future purchases made in the software market, then it would make sense for the term to be excluded from the flow utility of hardware. We choose the latter interpretation and exclude the indirect network effect from the flow utility, although it does enter the purchase period utility.

Note that unike most empirical research modeling two-sided markets, we structurally connect the hardware and software markets which both involve dynamic demand with forward-looking consumers, explicitly incorporating the utility of purchasing software into the hardware utility term. We detail explicitly the specific form of this connection between the markets in $\S 3.4$ below.

The price of console $j$ in period $t$ is denoted $p_{j, t}$. We capture seasonality in the hardware market with the month fixed effect term $\eta_{\tau(t)}^{h}$ where $\tau(t)=t \bmod 12$ represents the month of the year. The idiosyncratic shock specific to the consumer, console and period $\epsilon_{i j t}^{c}$ is unobservable and independently distributedas a Type I extreme value random variable, and is uncorrelated with all other unobservables in the model.

The coefficient $\alpha_{i}^{h}$ denotes the degree to which consumer $i$ values a console, whereas $\alpha^{x, h}$ indicates the effect of

\footnotetext{
${ }^{6}$ Similar to the software market, the consumer does not make the decision to purchase based only on the period utility. The decision-making process is detailed in 3.3
} 
consoles characteristics on the consumer's utility and finally $\alpha^{p, h}$ is the consumer's price coefficient. Note that the terms denoted by $v_{i j t}^{h}$ in the utility are deterministic persistent flow utility in the event of a purchase in period $t$. If consumer $i$ purchases the product $j$ in period $t$, then she exits the hardware market, and continues to receives a flow-utility in each period $\tau>t$ equal to $v_{i j t}^{h}$, which is fixed at the time of purchase.

\section{Bundles}

The above utility specification for hardware only considered consoles; however, in addition to consoles, consumers also have the choice to purchase a bundle of a console and a video game in periods when a bundle is available. We denote this selection by $\mathbf{B}_{\mathbf{t}}$, where a bundle $b \in \mathbf{B}_{\mathbf{t}}$ is represented as $b=(j, g)$, i.e. the bundle comprises of hardware console $j$ and software game $g$. In periods where there is no bundle available in the market, we set $\mathbf{B}_{\mathbf{t}}=\emptyset$ and consumers in those periods can only purchase consoles and games, i.e. pure components. Note that if the consumer purchases a bundle $b=(j, g)$, she exits the market for hardware (bundles and consoles).

When consumer $i$ considers the bundle option, the purchase period utility she derives from the purchase of bundle $b=(j, g)$ in period $t$ is given by $u_{i b t}^{b}$ :

$$
u_{i b t}^{b}=\overbrace{\underbrace{\alpha_{i}^{h}+\alpha^{x, h} x_{j t}}_{\text {Hardware Flow: } v_{i j t}^{h}-\xi_{j t}}+\underbrace{\alpha_{i}^{s}+\alpha^{w, s} w_{g t}}_{\text {Software Flow: } v_{i g t}^{s}-\chi_{g t}}+\mu_{b t}+\underbrace{\alpha^{b}}_{\text {Bundle Effect }}}^{\text {Total Bundle Flow: } v_{i b t}^{b}}+\psi W_{i t}^{b}+\alpha^{p, h} p_{b, t}+\underbrace{\eta_{\tau(t)}^{b}}_{\text {Seasonal Effects }}+\epsilon_{i b t}^{b}
$$

Thus, for consumer $i$, the utility of a bundle $b=(j, g)$ includes the deterministic components of the utility of console $j$, i.e. $v_{i j t}^{h}$ and the utility of the game that is included, $v_{i g t}^{s}$. Note that the unobservable terms from the console $j$ and video game $g$ corresponding to the bundle, $\xi_{j, t}$ and $\chi_{g, t}$ do not appear in the bundle utility function since they are product-specific unobservables and the bundle is positioned as a distinct product. Therefore, a bundlespecific unobservable utility $\mu_{b t}$ is included in the persistent flow utility. Note that $\mu_{b, t}$, the BLP-type unobservable characteristic of the bundle would include design, usability and other factors that may impact its utility above and beyond the utility of its constituent console and video game. We capture a generic effect of purchasing a bundle using $\alpha^{b}$ as the additional value that consumers have for the bundle over and beyond the value of the constituent hardware console and software game.

The idiosyncratic shock specific to the consumer, bundle and period, $\epsilon_{i b t}^{b}$ is again assumed to be distributed $i i d$ as Type I extreme value and uncorrelated with the other unobservables. We assume that $\mu_{b t}$ does persist beyond the period in which it is purchased, i.e. it appears in the flow utility of the bundle, and the terms denoted by $v_{i b t}^{b}$ in the utility are persistent "flow utility" in the event of a purchase. Thus, consumer $i$ who has purchased a bundle $b$ in period $t$ continues to receive a flow-utility of $v_{i b t}^{b}$ in period $\tau>t$, where the flow utility is fixed at the time of purchase. However, consistent with the model for consoles and for software, the bundle seasonal effect is not persistent in the model. As in the console model, we define the specific form of connection between the markets, 
or the indirect network effect, $W_{i t}^{b}$ in $\S 3.4$ below.

To complete the hardware model, we assume a consumer $i$ who is on the hardware market and decides not to purchase any hardware, console or bundle in period $t$ receives utility $\epsilon_{i, 0, t}$, while retaining the option to purchase hardware in future periods. Thus, the the vector of hardware error terms for consumer $i$ in period $t$ is $\epsilon_{\mathbf{i t}}^{\mathbf{H}}=$ $\left(\epsilon_{i 0 t}, \epsilon_{i, 1, t}^{c}, \ldots, \epsilon_{i,\left|J_{t}\right|, t}^{c}, \epsilon_{i, 1, t}^{b} \ldots, \epsilon_{i,\left|B_{t}\right|, t}^{b}\right)$, which are independently distributed across consumers, products and time periods.

Lastly, we fix consumers' price sensitivity to be equal for hardware and software, i.e. $\alpha^{p, s}=\frac{\alpha^{p, h}}{\psi}$. From a structural viewpoint, the price coefficient for consumers might be different for hardware and software only when the purchase of a hardware console would cause a change in wealth, leading to a change in price-sensitivity 7 We note that consumer heterogeneity enters through the preference for consoles and gaming in general (a hardware constant and software constant), and all other coefficients are homogeneous in our model specification.

\subsection{Consumer's Decision Problem}

We now describe consumer decisions in chronological order, beginning with the hardware market, followed by those in the software market.

\subsubsection{Hardware Market Decisions}

Consumers in the hardware market in period $t$ purchase a console or bundle after evaluating utilities from options available in that period and forming expectations over the evolution of both hardware and software markets. When a consumer purchases a bundle or console, she exits the hardware market and does not return to it in future periods, and she enters the software market. Given this setup, the decision problem is inherently dynamic, and the consumer faces a choice of when to purchase hardware.

Consider consumer $i$ 's decision problem for the console or bundle in a specific period, $t$ : she has to decide whether to buy a hardware product now or wait until the next period to make a similar decision. In order to account for the value of waiting, followed by adoption at some point in the future, the consumer has to anticipate the evolution of all variables $\Omega_{i, t}$ that will affect the value of the future adoption decision. The state $\Omega_{i, t}$ ideally ought to include the future evolution of console characteristics (both observable and unobservable), entry of new hardware, i.e. consoles or bundles, future price trajectory, as well the video games that might be available in the future software market, since each of these variables might affect the utility of a future hardware adoption.

For consumer $i$, the Bellman equation that describes her value for being in a current state $\Omega_{i, t}^{h}$ in the hardware

\footnotetext{
${ }^{7}$ We ought to expect this effect to be very small in our setting, given the low price of consoles, relative to average income levels; indeed, our results when using different price coefficients for hardware and software are quantitatively very similar and qualitatively the same as with the primary specification.
} 
market is given by the recursive relationship:

$V_{i}^{h}\left(f_{i t}, \Omega_{i, t}^{h}, \epsilon_{\mathbf{i t}}^{\mathbf{h}}\right)=\max \{\underbrace{\max _{h \in \mathbf{H}_{\mathbf{t}} \backslash\{\mathbf{0}\}}\left\{u_{i h t}\left(\epsilon_{i h t}\right)+\beta E\left[V_{i}\left(v_{i h t}^{h}, \Omega_{i, t+1}^{h}, \epsilon_{\mathbf{i}, \mathbf{t}+\mathbf{1}}^{\mathbf{h}} \mid \Omega_{i t}^{h}\right)\right]\right\}}_{\text {Purchase hardware } h}, \underbrace{\beta \mathbf{E}\left[V_{i}^{h}\left(f_{i t}, \Omega_{i, t+1}^{h}, \epsilon_{\mathbf{i}, \mathbf{t}+\mathbf{1}}^{\mathbf{h}} \mid \Omega_{i, t}\right)\right]+\epsilon_{i 0 t}^{h}}_{\text {No Purchase }}\}$

The first term represents the case when the consumer makes a purchase of a console or bundle in period $t$, whereas second corresponds to the 'no purchase' option, where the consumer defers the decision to the next period. The value function $V_{i}^{h}$ corresponding to the current state variable $\Omega_{i, t}^{h}$ then represents the maximum utility from these two possibilities, i.e. no purchase or purchase. $V_{i}^{h}$ depends on the current flow utility $f_{i t}$ (derived from a currently owned hardware product) state variable $\Omega_{i, t}^{h}$ and the idiosyncratic shocks experienced by the consumer in the hardware market.

Note that the above is a general enough to apply to the case when consumers can replace their hardware. When consumers do not replace hardware, but exit the hardware market upon making a purchase, we can simplify the second term in the Bellman equation to the stream of flow utilities obtained from the purchase of hardware $h$ (console or bundle), which is $\left(\sum_{\tau=t+1}^{\infty} \beta^{\tau-t} v_{i h t}^{h}\right)$. Note that it is independent of $\Omega_{i, t+1}^{h}$ and $\epsilon_{\mathbf{i}, \mathbf{t}+\mathbf{1}}^{\mathbf{h}}$ as well as other future terms. If the consumer exits the hardware market upon purchase, the current flow $f_{i t}$ can be dropped from the value function, leading to the following Bellman equation:

$$
V_{i}^{h}\left(\Omega_{i, t}^{h}, \epsilon_{\mathbf{i t}}^{\mathbf{h}}\right)=\max \left\{\max _{h \in \mathbf{H}_{\mathbf{t}} \backslash\{\mathbf{0}\}}(\underbrace{u_{i h t}\left(\epsilon_{i h t}^{h}\right)}_{\text {Period Utility }}+\underbrace{\frac{\beta}{1-\beta}\left(v_{i h t}^{h}\right)}_{\text {Future Stream of Flow Utility }}), \beta \mathbf{E}\left[V_{i}^{h}\left(\Omega_{i, t+1}^{h}, \epsilon_{\mathbf{i}, \mathbf{t}+\mathbf{1}}^{\mathbf{h}} \mid \Omega_{i, t}^{h}\right)+\epsilon_{i 0 t}^{h}\right]\right\}
$$

where the period utility $u_{i h t}$ and the flow utility $v_{i h t}^{h}$ are obtained from equations (3) and (5). The expected value function is obtained by integrating out the error terms in the value function:

$$
E V_{i}^{h}\left(\Omega_{i, t}^{h}\right)=\int V_{i}^{h}\left(\Omega_{i, t}^{h}, \epsilon_{\mathbf{i t}}^{\mathbf{h}}\right) d F_{\epsilon^{\mathbf{h}}}\left(\epsilon_{i t}^{h}\right)
$$

where $F_{\epsilon^{\mathrm{h}}}$ is the distribution function corresponding to $\epsilon^{\mathbf{h}}$ (Type I Extreme Value).

Hardware Inclusive Value The inclusion of all relevant variables within $\Omega_{i, t}^{h}$ is clearly problematic from the viewpoint of tractability, in the sense that the state space of the dynamic problem grows exponentially, and solving for the value function is computationally infeasible. Moreover, the researcher does not observe all the information that consumers and firms possess that might affect future expectations. Given these considerations, we follow the idea of inclusive value introduced by Melnikov 2001 and developed further by others Hendel and Nevo, 2006, Gowrisankaran and Rysman, 2013. 
The inclusive value represents the "expected utility of the best purchase option," and is intended to tractably capture the effects of all state variables in $\Omega_{i, t}^{h}$ that affect future utility into a consumer-specific inclusive-value state variable $\delta_{i t}$. The rationale is that there may be multiple changes in the future, i.e. products may increase in quality, prices may decline, new products may become available etc. However, the effect of all these factors would be to increase or decrease the expected utility of the best purchase option in the future, or the inclusive value. Thus, if we know how the inclusive value evolves over time, we can capture all the key drivers of the consumer's decision making process. The inclusive value sufficiency (IVS) assumption implies that $\delta_{i t}^{h}$ would be "sufficient" to capture the variation in $\Omega_{i t}^{h}$ for the purposes of a consumer's decision making process. Note that while $\Omega_{i t}^{h}$ is a possibly large-dimensioned vector that includes expectations about how new products may be introduced, prices may increase or decrease, old products might be withdrawn from the marketplace etc. IVS is the bridge that allows us to transform the large-dimensional $\Omega_{i t}^{h}$ space into the single dimensional $\delta_{i t}^{h}$ space.We discuss further details of the inclusive value specification and provide empirical support for its appropriateness in Appendix B.

This inclusive value simplification ensures that the state space is tractable, and we assume that the individual consumer's inclusive value is sufficient to represent choice probabilities, dramatically reducing the state space to one dimension. The expected utility from each purchase option for consoles and bundles depends on both the flow utilities and the effects specific to the purchase period, and can be characterized as:

$$
\delta_{i k t}^{h}= \begin{cases}\frac{v_{i k t}^{h}}{1-\beta}+\alpha^{p, h} p_{k t}+\eta_{\tau(t)}^{h}, & k \in \mathbf{J}_{\mathbf{t}} \\ \frac{v_{i k t}^{b}}{1-\beta}+\alpha^{p, h} p_{k t}+\eta_{\tau(t)}^{b}, & k \in \mathbf{B}_{\mathbf{t}}\end{cases}
$$

Note that $v_{i j t}^{h}$ and $v_{i b t}^{b}$ are the flow utilities for consoles and bundles and the present discount value of the flow utility is thus equal to $\frac{v_{i k t}^{h}}{1-\beta}$ or $\frac{v_{i k t}^{b}}{1-\beta}$. The inclusive value $\delta_{i t}^{h}$ is then defined based on the inclusive utilities to represent the expected value of purchasing any of the hardware options (consoles or bundles) as:

$$
\delta_{i t}^{h}=\mathbf{E}_{\epsilon_{\mathbf{i t}}^{\mathrm{h}}}\left[\max _{k \in \mathbf{J}_{\mathbf{t}} \cup \mathbf{B}_{\mathbf{t}}}\left(\delta_{i k t}^{h}+\epsilon_{i k t}^{h}\right)\right]=\log \left(\sum_{k \in \mathbf{J}_{\mathbf{t}} \cup \mathbf{B}_{\mathbf{t}}} \exp \left(\delta_{i k t}^{h}\right)\right) .
$$

The Bellman equation can consequently be expressed in terms of the hardware inclusive value, $\delta_{i t}^{h}$ :

$$
E V_{i}^{h}\left(\delta_{i t}^{h}\right)=\log (\underbrace{\exp \left(\delta_{i t}^{h}\right)}_{\text {Purchase }}+\underbrace{\exp \left(\beta \mathbf{E}\left[E V_{i}\left(\delta_{i, t+1}^{h}\right) \mid \delta_{i, t}^{h}\right]\right)}_{\text {No Purchase }})
$$

We model the inclusive value $\delta_{i, t}^{h}$ to be perceived by the consumer evolving according to an $A R(1)$ process:

$$
\delta_{i, t+1}^{h}=\gamma_{i, 0}^{h}+\gamma_{i, 1}^{h} \delta_{i, t}^{h}+\zeta_{i, t}^{h}
$$


where $\zeta_{i, t}^{h}$ is distributed as a standard normal and is iid across consumers and time periods. The individual-specific parameters $\gamma_{i, 0}^{h}$ and $\gamma_{i, 1}^{h}$ characterize the evolution of the inclusive value state, and yield a probability distribution for the future state, conditional on the current state.

The expected value functions $E V_{i}^{h}$ from the Bellman equation are used to obtain the conditional purchase probabilities for consumers. Consumer $i$ 's probability of purchasing product $k$ is given as a function of the inclusive value in the corresponding period, $\delta_{i t}^{h}$ as follows:

$$
\hat{s}_{i k}^{h}\left(\delta_{i t}^{h}\right)=\frac{\exp \left(\delta_{i t}^{h}\right)}{\left[\exp \left(E V_{i}^{h}\left(\delta_{i t}^{h}\right)\right)\right]} \frac{\exp \left(\delta_{i k t}^{h}\right)}{\exp \left(\delta_{i t}^{h}\right)} .
$$

The first fraction represents the probability of purchase for consumer $i$ and the second represents the probability of choosing alternative $k$, conditional on deciding to make a purchase. The inclusive value this separates out the probability from making a purchase from the probability of purchasing a specific product conditional of making a purchase - this feature is important because the latter term does not depend on dynamic factors in the hardware market and can be easily computed.

After a consumer makes a hardware purchase, she exits the hardware market and enters the software market, and we examine the decision making process in that market next.

\subsubsection{Software Market Decisions}

The consumer's decision problem for software is somewhat different from the hardware decision described above. Consumers in each period face the choice of purchasing a software video game title, or making no purchase at all. However, the decision making process in the software market differs conceptually from that of hardware along two different dimensions:

- Consumers do not exit after they make a purchase in the software market, but continue to make further software purchases over time as illustrated in Figure 3.

- When a consumer purchases a new video game software title, it does not replace previously owned video games, but adds to the consumer's portfolio of software, all of which continue to provide flow utility 8 Thus in period $t$, the consumer's software flow utility $F_{i t}^{s}$ accrues from past purchases and is represented as:

$$
F_{i t}^{s}=\left(\sum_{\tau=1}^{t-1} \sum_{g \in \mathbf{S}_{\tau}} v_{i g \tau}^{s} \quad \mathbf{1}\left\{d_{i g \tau}=1\right\}\right)
$$

We define $F_{i t}^{s}$ as the flow utility at the beginning of period $t$, so that purchases made during period $t$ are not

8

- In the hardware market, replacements consoles are often modeled as making older consoles owned by consumers irrelevant. 
included until the following period.

Similar to the hardware market, the state variables affecting the current and future utility of consumers are represented by $\Omega_{i, t}^{s}$. In the software market, when a consumer adds an additional software title, her incremental utility depends on her current software holdings (of $N_{g}$ games before the current purchase), and is modeled by the declining marginal utility of holding multiple games, denoted by the function $\varphi$.

The discounted flow utility associated with the software purchase is $\sum_{\tau=t}^{\infty} \beta^{\tau-t} v_{i g t}^{s}=\frac{v_{i g t}^{s}}{1-\beta}$, and is incorporated into the period utility when the consumer is considering a purchase. The price effect in the second term and the seasonal effect in the third term are only in effect during the purchase period and not beyond that time period. Note that the consumer is modeled as having a decreasing marginal utility for games, represented by the function $\varphi$ in $N_{g}$, the number of game titles already owned by the consumer before making the current purchase. The final term represents the expected value function of continuing to the future after making a purchase of game $g$ in the current period, and thus holding $\left(N_{g}+1\right)$ games when entering the next period.

The Bellman equation corresponding to the software market is consequently specified in terms of the state variables representing overall evolution of the software market $\left(\Omega_{i, t}^{s}\right)$, flow utility from the consumer's current software portfolio $\left(U_{i 0 t}\right)$, and the number of games owned by the consumer $\left(N_{g}\right)$.

$$
\begin{gathered}
E V_{i}^{s}\left(\Omega^{s}, F_{i t}^{s}, N_{g}\right)=F_{i t}^{s}+\mathbf{E}_{\epsilon^{\mathrm{s}}}\left[\max \{\overbrace{\max _{g \in \mathbf{S}_{\mathbf{t}}}\left(u_{i g t}\left(\epsilon_{i g t}^{s}\right)-\varphi\left(N_{g}+1\right)+\beta \mathbf{E}_{\Omega^{s}}\left[E V_{i}^{s}\left(F_{i t}^{s}+v_{i g t}^{s}, \Omega_{i, t+1}^{s}, N_{g}+1\right) \mid \Omega_{i, t}^{s}\right]\right)}^{\text {Purchase any available video game }},\right. \\
\underbrace{\epsilon_{i 0 t}^{s}-\varphi\left(N_{g}\right)+\beta \mathbf{E}_{\Omega^{s}}\left[E V_{i}^{s}\left(F_{i t}^{s}, \Omega_{i, t+1}^{s}, N_{g}\right) \mid \Omega_{i, t}^{s}\right]}_{\text {No Purchase }}\}]
\end{gathered}
$$

The first term in the above expectation represents the value of buying and continuing to hold software while the second term is the value of not buying and continuing to hold software games. Observe that the consumer's current flow from her software portfolio, $F_{i t}^{s}$, is additive across all options, and does not impact the decision directly; rather the decision depends on the number of games owned by the consumer. This feature of the problem enables us to simplify the characterization of the consumer's decision as depending on the number of games, rather than specific games owned by the consumer. The intuitive observation that two consumers with identical preferences, beginning with different levels of flow utility, say $F_{i t}^{s}=f$ and $F_{i t}^{s}=f^{\prime}$ will make the same decisions, conditional on having the same number of games (say $N_{g}$ ) 9

\footnotetext{
${ }^{9}$ This can be easily proven, similar to the cases in Hendel and Nevo 2006], Gowrisankaran et al. 2010, by considering the following transformation: $E V_{i}^{s}\left(\Omega^{s}, N_{g}\right)=E V_{i}^{s}\left(\Omega^{s}, F_{i t}^{s}, N_{g}\right)-\frac{F_{i t}^{s}}{1-\beta}$ which when substituted in the Bellman equation 14 gives us:

$$
\begin{aligned}
& E V_{i}^{s}\left(\Omega^{s}, N_{g}\right)+\frac{F_{i t}^{s}}{1-\beta}=F_{i t}^{s}+\mathbf{E}_{\epsilon^{\mathbf{s}}}\left[\operatorname { m a x } \left\{\max _{g \in \mathbf{S}_{\mathbf{t}}}\left(u_{i g t}\left(\epsilon_{i g t}^{s}\right)-\varphi\left(N_{g}+1\right)+\beta \mathbf{E}_{\Omega^{s}}\left[\frac{\left(F_{i t}^{s}+v_{i g t}^{s}\right)}{1-\beta}+E V_{i}^{s}\left(\Omega_{i, t+1}^{s}, N_{g}+1\right) \mid \Omega_{i, t}^{s}\right]\right)\right.\right. \\
& \left.\left.\epsilon_{i 0 t}^{s}-\varphi\left(N_{g}\right)+\beta \mathbf{E}_{\Omega^{s}}\left[\frac{F_{i t}^{s}}{1-\beta}+E V_{i}^{s}\left(\Omega_{i, t+1}^{s}, N_{g}\right) \mid \Omega_{i, t}^{s}\right]\right\}\right]
\end{aligned}
$$

where upon simplification the terms involving $F_{i t}^{s}$ cancel out giving us the simplified software market Bellman equation 15 . 
Hence, we can just write the simplified expected value function without the flow utility in the software market as:

$$
\begin{gathered}
E V_{i}^{s}\left(\Omega^{s}, N_{g}\right)=\mathbf{E}_{\epsilon^{\mathrm{s}}}\left[\max \{\overbrace{\max _{g \in \mathbf{S}_{\mathbf{t}}}\left(u_{i g t}\left(\epsilon_{i g t}^{s}\right)+\frac{\beta}{1-\beta} v_{i g t}^{s}-\varphi\left(N_{g}+1\right)+\mathbf{E}_{\Omega^{s}}\left[E V_{i}^{s}\left(\Omega_{i, t+1}^{s}, N_{g}+1\right) \mid \Omega_{i, t}^{s}\right]\right.}^{\text {Purchase any available video game }},\right. \\
\underbrace{\epsilon_{i 0 t}^{s}-\varphi\left(N_{g}\right)+\mathbf{E}_{\Omega^{s}}\left[E V_{i}^{s}\left(\Omega_{i, t+1}^{s}, N_{g}\right) \mid \Omega_{i, t}^{s}\right]}_{\text {No Purchase }}\}]
\end{gathered}
$$

where the consumer can be thought of as obtaining the present discounted flow utilities from the game $\left(\frac{\beta}{1-\beta} v_{\text {igt }}^{s}\right)$ instantaneously upon purchase.

Modeling The Software Portfolio Given the situation that all we cannot tractably track the entire software portfolio of the consumer, we face trade-offs in modeling the consumer decision making process. First, as an approximation, we can track the number of titles purchased by each consumer, and update that each period. However, with this approach, we would not be able to dynamically alter the choice set for each consumer based on prior purchase decisions made by that consumer. The downside is that consumers might purchase the same game multiple times over several periods, which we might believe to be less likely to happen in reality. Thus, in some cases consumers might purchase the same game twice, although we might not expect this to happen frequently for two reasons: (a) consumers in general have a low purchase probability for any game title, given that there are hundreds of titles. (b) software titles reach their peak pretty early in their life-cycle and decline in sales beyond that, so if a consumer hasn't found a high-enough utility in an early period, she's not likely to obtain a high utility in later periods. Keeping these considerations in mind, we set the choice set in any period $t$ to be equal to all software titles available for sale in period $t$, denoted by $\mathbf{S}_{\mathbf{t}}$, even though the actual choice set for the consumer would be arguably smaller and based on past purchases. We however capture the diminishing marginal utility from software using the number of game titles owned by the consumer in the utility formulation.

As an alternative, we might model separately the market for each product, implicitly assuming that software titles are local monopoly markets. We could in this case track the number of consumers who have so far purchased the software, and dynamically update the potential market to include the number of households who have not yet purchased the product. However, we would not be able to track the competitive interactions between different software titles, since we would have to assume that consumers make separate decisions in the separate market for each software title. We note that the results are qualitatively similar under these two market conditions, lending support to the robustness of our findings 10 Observe that the true market reality has partial aspects of both of these modeling options, and since these approaches represent extreme possibilities, we would expect them to enclose the essential features of the market.

\footnotetext{
${ }^{10}$ Results for this model specification are available from the authors upon request.
} 
Inclusive Value in Software Market There are two primary sources of dynamic variation in consumer utility that we must capture with the idea of inclusive value: (a) the consumer's software portfolio of games, and (b) the industry dynamics of the flow utility obtained from making a purchase in the software market.

We collapse all the factors that might affect the industry evolution $\Omega^{s}$ into an evolving inclusive value $\omega^{s}$, which is different from the case of the hardware market. We do this to simplify and separate out the evolution of the industry from the evolution of the consumer's portfolio, as she adds games by making purchases over time. Note that this evolving inclusive value term could be interpreted as the expected stream of utilities from best purchase option, independent of the software portfolio held by any consumer. We define the evolving inclusive value term for consumer $i$ in period $t, \omega_{i t}^{s}$ below and its evolution by an $\operatorname{AR}(1)$ process as follows:

$$
\begin{gathered}
\omega_{i t}^{s}=\log \left(\sum_{g \in \mathbf{S}_{\mathbf{t}}} \exp \left(\frac{v_{i g t}^{s}}{1-\beta}+\alpha^{p, g} p_{g t}+\eta_{\tau(t)}^{s}\right)\right) \\
\omega_{i t+1}^{s}=\gamma_{i, 0}^{s}+\gamma_{i, 1}^{s} \omega_{i t}^{s}+\zeta_{i, t}^{s}
\end{gathered}
$$

The utility of purchasing game $g$ in the inclusive value framework can then be defined net of the error term in terms of the evolving inclusive value:

$$
\delta_{i g t}^{s}=\left(\frac{v_{i g t}^{s}}{1-\beta}+\alpha^{p, g} p_{g t}+\eta_{\tau(t)}^{s}\right)-\varphi\left(N_{g}+1\right)+\beta \mathbf{E}_{\omega_{\mathrm{i}}}\left[E V_{i}^{s}\left(\omega_{i, t+1}^{s}, N_{g}+1\right) \mid \omega_{i, t}^{s}\right]
$$

Similar to the hardware market, we attempt to capture the software inclusive value, $\delta_{i t}^{s}$, which is the expected utility of the best purchase option in period $t$. This inclusive value would also track the consumer's software portfolio (of $N_{g}$ games), and incorporate the variation captured by the evolving inclusive value. The software inclusive value $\delta_{i t}^{s}$ representing the expected maximum utility from making a purchase and continuing in the software market includes the evolving inclusive value as well the consumer's current software portfolio of $N_{g}$ games and is defined as follows:

$$
\begin{gathered}
\delta_{i t}^{s}\left(N_{g}\right)=\log \left(\sum_{g \in \mathbf{S}_{\mathbf{t}}} \exp \left(\left[\frac{v_{i g t}^{s}}{1-\beta}+\alpha^{p, g} p_{g t}+\eta_{\tau(t)}^{s}\right]-\varphi\left(N_{g}+1\right)+\beta \mathbf{E}_{\omega_{\mathbf{i}}}\left[E V_{i}^{s}\left(\omega_{i, t+1}^{s}, N_{g}+1\right) \mid \omega_{i, t}^{s}\right]\right)\right) \\
=\omega_{i t}^{s}-\varphi\left(N_{g}+1\right)+\beta \mathbf{E}_{\omega_{\mathbf{i}}}\left[E V_{i}^{s}\left(\omega_{i, t+1}^{s}, N_{g}+1\right) \mid \omega_{i, t}^{s}\right]
\end{gathered}
$$

because the last two terms in equation (19) are constant across all games.

The evolving inclusive value is designed to capture the changes that are occuring in the market, and the expectation that the consumer has about how these changes will affect the future expected value of the software market. Separating out the evolving inclusive value (which varies based on factors largely exogenous to the consumer), from the dynamics of how consumers add to their software portfolio allows us to tractably characterize the software market. Ideally, we would model the consumer's entire software portfolio instead of capturing it with the number 
of games owned by the consumer; however, the state space would explode in this setting and make the problem intractable, and we therefore choose to approximate the portfolio by the number of games. The separation of the industry evolution captured by the evolving inclusive value, and the consumer's portfolio evolution captured by the software inclusive value also enables the computation to be tractably carried out in a setting with a large number of software choices and heterogeneous consumers.

We rewrite the Bellman equation in terms of the evolving inclusive value as:

$$
\begin{aligned}
E V_{i}^{s}\left(\omega_{i t}^{s}, N_{g}\right)=\log \{ & \underbrace{\exp \left(\omega_{i t}^{s}-\varphi\left(N_{g}+1\right)+\beta \mathbf{E}_{\omega}\left[E V_{i}^{s}\left(\omega_{i, t+1}^{s}, N_{g}+1\right) \mid \omega_{i t}^{s}\right]\right)}_{\text {Purchase }} \\
& +\underbrace{\exp \left(-\varphi\left(N_{g}\right)+\beta \mathbf{E}_{\omega}\left[E V_{i}^{s}\left(\omega_{i, t+1}^{s}, N_{g}\right) \mid \omega_{i t}^{s}\right]\right)}_{\text {No Purchase }}\}
\end{aligned}
$$

As detailed in the discussion following equation 14, we can eliminate $U_{i 0 t}$ as a state variable from the Bellman equation, since it affects both the value of purchasing a new software title and of not purchasing. After determining the expected value function, we then determine the conditional probability of purchasing a specific software title $g$. For consumer $i$, the probability of making any purchase in period $t$ is represented by $P_{i t}^{s}\left(\delta_{i t}^{s}, N_{g}\right)$ as:

$$
P_{i t}^{s}\left(\delta_{i t}^{s}, N_{g}\right)=\frac{\exp \left(\delta_{i t}^{s}\left(N_{g}\right)\right)}{\exp \left(E V_{i}^{s}\left(\delta_{i t}^{s}, N_{g}\right)\right)}
$$

Conditional on purchasing, the consumer chooses game $g$ in period $t$ with probability

$$
P_{i g t}^{s}=\exp \left(\delta_{i g t}^{s}-\delta_{i t}^{s}\right)=\exp \left(\frac{v_{i g t}^{s}}{1-\beta}+\alpha^{p, g} p_{g t}+\eta_{\tau(t)}^{s}-\omega_{i t}^{s}\right)
$$

To find the unconditional purchase probability for game $g$, we must determine the fraction of consumers of type $i$ holding $N_{g}$ number of games in period $t$. Denote this proportion as $\Lambda_{i t}\left(N_{g}\right)$. With this we can determine the unconditional purchase probability of (forward-looking) consumers of type $i$ purchasing software $g$ in period $t$ as:

$$
s_{i g t}^{\text {dynamic }}=P_{i g t}^{s}\left(\sum_{N_{g}=0}^{N_{\max }} P_{i t}^{s}\left(\delta_{i t}^{s}, N_{g}\right) \Lambda_{i t}\left(N_{g}\right)\right),
$$

where $N_{\max }$ represents the number of games held by a consumer beyond which the utility effects are modeled to be identical and aggregated in the state space of the model. Again, this maximum ensures that we can tractably capture the diminishing marginal effect of software titles.

Comparison of Hardware and Software Inclusive Value It is worthwhile asking the question what exactly the evolving and total software inclusive value represent, and how the software inclusive value is different from 
the hardware inclusive value. We first discuss what is common to the notion of inclusive value in both hardware and software markets. The inclusive value (in both markets) is expected to be higher when: (a) there are more products available for sale (giving the consumer better options to purchase), (b) when the price of any product is lower (giving the consumer more surplus from any purchase), (c) the mix of products is newer and higher value, and (d) products have higher unobserved product quality. All of the above factors affect the expected utility best purchase option for the consumer in a specific period, and hence the inclusive value in both markets.

In the software market, the consumer continues in the market by either making a purchase and adding to her software portfolio, or not making a purchase and obtaining the flow utility from the current portfolio (i.e. with no addition). This feature of the setting results in a few crucial differences between the inclusive value in the hardware market and in the software market. First and most important, in the hardware market, the inclusive value determines the flow utility that a consumer obtains from a purchase (less the price effect and seasonality), since a new purchase also implies exit from the hardware market 11 However, in the software market, this relationship between inclusive value and flow utility does not hold. A consumer's current flow utility derives from a number of games held by the consumer, whereas the inclusive value represents the best purchase (i.e. one software title), which only adds to the portfolio and thus adds to the flow utility of the consumer, rather than replacing it as in hardware. Note that although we use the number of software titles as a sufficient statistic for current holdings, the software purchase decision carefully accounts for the game characteristics, price and unobservable utility.

There are other subtle issues which result in the hardware and software inclusive values being different: consumers in the software market are not often replacing older software, a price decrease of a game that is not the newest and latest software title can still have a significant positive impact on the inclusive value. Similarly, the introduction of a new product might not change the inclusive value to the same degree that we might observe in the hardware market, given that there are a large number of titles and the products are more likely to be horizontally differentiated.

\section{Seasonal Myopic Consumers}

Given that there are large seasonal spikes in software sales during the holiday season months of November and December, we enrich the model by allowing the entry of myopic gift givers into the software market during these months. We observe that the average number of games sold per hardware owner in November or December is more than one, i.e. more games are sold than there are console owners. Thus, we model the entry of myopic consumers due to two underlying reasons. First, we recognize the institutional importance of holiday gift giving in the video game market, where the product is positioned at children. Second, this allows the model more flexibility to match the reality of consumer decisions in the market more accurately. Myopic consumers have the same marginal utilities

\footnotetext{
${ }^{11}$ Even in the case of repeat purchases, this would hold since the inclusive value would represent a replacement of the current flow utility with the new purchased product's flow utility.
} 
toward game characteristics as the forward looking (or dynamic) consumers we have modeled above, but they do not look into the future to form expectations or make intertemporal tradeoffs when making purchase decisions. We account for these consumers in our model by characterizing the potential market size for software to be larger by $50 \%$ during the months of November and December than it would be in their absence. The market share for game $g$ in period $t$ in the software market is then given by:

$$
\hat{s}_{g t}=\left[1-\mathbf{I}\left(\text { seasonal }_{t}\right)\right] \sum_{i \in \mathbf{I}_{\mathbf{t}}} \lambda_{i, t} s_{i g t}^{\text {dynamic }}+\mathbf{I}\left(\text { seasonal }_{t}\right)\left[\frac{2}{3} \sum_{i \in \mathbf{I}_{\mathbf{t}}} \lambda_{i, t} s_{i g t}^{\text {dynamic }}+\frac{1}{3} \sum_{i \in \mathbf{I}_{\mathbf{t}}} \lambda_{i, t} s_{i g t}^{\text {myopic }}\right]
$$

where $\lambda_{i, t}$ is the fraction of consumers of type $i$ in period $t, \mathbf{I}\left(\right.$ seasonal $\left._{t}\right)$ is an indicator variable for the periods corresponding to the months of November or December and $s_{i g t}^{\text {myopic }}$ is the probability of myopic consumer of type $i$ purchasing game $g$ in period $t$. $s_{i g t}^{\text {myopic }}$ then takes the form:

$$
s_{i g t}^{\text {myopic }}=\frac{\exp \left(v_{i g t}^{s}+\alpha^{p, g} p_{s, t}+\eta_{\tau(t)}^{s}\right)}{1+\left(\sum_{g \in \mathbf{S}_{\mathbf{t}}} \exp \left(v_{i g t}^{s}+\alpha^{p, g} p_{s, t}+\eta_{\tau(t)}^{s}\right)\right)} .
$$

\subsection{Linking Hardware and Software: Indirect Network Effect}

We structurally connect the utilities for the consumer in the hardware and software markets through the indirect network effect. The intuition is that consumers have an expectation over the evolution of the software market and the value provided by software in determining whether to make a hardware purchase. Thus, the expected value of being present in the software market, which is opened up by making a hardware purchase is a key driver of hardware utility. Referring to the hardware (console or bundle) period utility model in $\$ 3.2$ above, the connection represented by $W_{i t}^{c}$ in equation (3), the console period utility function, is also defined in terms of the inclusive value. Specifically, it is the expected value function for the software model in period $t$ for consumer $i$, and thus accounts for the future evolution of the software market, including changes in software availability (e.g. new games) as well as pricing dynamics.

$$
W_{i t}^{c}=E V_{i}^{s}\left(\omega_{i t}^{s}, N_{g}=0\right)
$$

Note the term $W_{i t}^{b}$ linking the bundle utility and the software market utility is defined as

$$
W_{i t}^{b}=E V_{i}^{s}\left(\omega_{i t}^{s}, N_{g}=1\right)
$$

and is contingent on the fact that the consumer will have one game in his portfolio when entering the software market. Note that the above terms represent the indirect network effect at the time of purchase. However, after purchase the network effect would be expected to increase e.g. due to more software titles becoming available. In our rational expectations framework, the consumer would have an expectation that more titles would become 
available after purchase, but if the future realization is that the software market is more attractive for consumers than expected, the consumer would realize a higher post-purchase utility. However, such an effect would not be identified since the consumer has already made a purchase, and we cannot include such an effect in our model.

\subsection{Heterogeneity}

We specify consumers to be heterogeneous in their value for hardware $\left(\alpha_{i}^{h}\right)$ and their value for software $\left(\alpha_{i}^{s}\right)$. The specification leads to consumer-specific indirect network effects, denoted by $W_{i t}$, but consumers are homogenous in how they value the characteristics of hardware $\left(\alpha^{x, h}\right)$ and software $\left(\alpha^{x, s}\right)$, as well as price $\left(\alpha^{p, h}\right)$. We discuss the nature of identification issues and detail the reasons for this modeling choice in 4 The connection between the dimensions of heterogeneity is captured by characterizing consumer $i$ 's value for hardware $\alpha_{i}^{h}$ and for software $\alpha_{i}^{s}$ to be jointly distributed normal random variables as follows:

$$
\left(\begin{array}{c}
\alpha_{i}^{s} \\
\alpha_{i}^{h}
\end{array}\right) \sim N\left(\left(\begin{array}{c}
\bar{\alpha}^{s} \\
\bar{\alpha}^{h}
\end{array}\right), \boldsymbol{\Sigma}\right), \quad \text { where } \boldsymbol{\Sigma}=\left(\begin{array}{cc}
\sigma_{s}^{2} & \rho \sigma_{s} \sigma_{h} \\
\rho \sigma_{s} \sigma_{h} & \sigma_{h}^{2}
\end{array}\right)
$$

Notice that the above specification generalizes the standard practice of assuming preferences for products across multiple categories to be independent, used in all of the dynamic demand literature. We allow the data to determine the correlation between console and gaming preferences, and do not impose a specific form of dependence. We include this dependence to allow better flexibility in representing purchase patterns across the software and videogame markets and more importantly, to characterize the homogenization effect, i.e. the degree by which bundling reduces heterogeneity of consumer valuations and helps in extracting surplus.

\section{Identification and Estimation}

We have previously developed a model of consumer demand in a dynamic durable goods platform market for a monopolist firm with multiple hardware consoles, and a software market characterized by a variety of video games produced over time. Consumers in this market face several decisions, ranging from which hardware to purchase, and when to make a purchase, as well as determining which software game title to purchase in each period after they own a console. We next discuss how the parameters of the consumer utility model are identified using the variation in the data from the handheld videogame industry, and detail the estimation process for our dynamic demand model using aggregate sales data on consoles, videogames, and bundles.

\section{Identification}

We discuss the identification of the parameters in the above model to help understand what variation in the data permits the estimation of each of the parameters. First, consider the consumer utility for video game software, 
$\alpha_{i}^{s}$ : a higher value of this coefficient implies more game sales, other factors being the same. The heterogeneity of consumer preferences for software $\left(\sigma_{s}\right)$ is identified by the rate of increase (or decrease) in sales of each software title over time. Consider the extreme case with no heterogeneity, in which case the only difference between consumers is due to the error term. In such a case, the sales spike would be sharp both upward and downward compared to the case with significant heterogeneity, where we would find the sales rate increase (or decrease) to be more gradual. Note that we consider heterogeneity in consumer valuation for consoles and software (the intercept term), rather than heterogeneity in price sensitivity. We focus on the above dimensions of heterogeneity since one of our key objectives is to understand the effect of correlation in consumer valuation across products that comprise a bundle. We would be unable to capture these bundling effects if we had chosen to focus on heterogeneity in the coefficient of price.

Next, consider the game characteristics, which are all dynamically varying: age, and higher powers of age determine the variation of sales with product characteristics, and identifies the coefficient $\alpha^{w, s}$. Average effects of these time trends identify these parameters, whereas the game-specific unobservable $\chi_{g t}$ for game $g$ in period $t$ rationalizes market sales. The price coefficient $\alpha^{p, s}$ is identified as usual by dynamic variation in price levels and sales levels.

In the hardware market, the product characteristics coefficients $\alpha^{x, h}$ are identified by variations in console characteristics over time, both from the variation of console sales and prices across markets (time periods in our setting), and from whether increased sales for a specific product come from other products that are "more similar" or "less similar" in terms of product characteristics. While these previous sources of variation are the basic elements in BLP and much of the literature, our setting provides an additional source of variation resulting from the explicit dynamic model that incorporates intertemporal trade-offs, that enables another source of support for identification. More specifically, if consumers with the highest value for hardware $\left(\alpha_{i}^{h}\right)$ purchase earlier and exit the hardware market, it would imply that consumers who remain have a lower valuation for hardware. This dynamic effect along with the change in product characteristics of hardware over time thus serves as an additional source of variation.

The identification for consumer heterogeneity in preference for hardware $\left(\sigma_{h}\right)$ is similar to that of software, but the variation in the availability of bundles over time provides an additional source for its identification, because it allows the rate of increase (and decrease) of bundle sales to be used for this purpose. Note that the unobservable product-period specific shocks to consoles and games also carry over to the bundles that include them. The identification of the normalization parameter $\psi$ that permits direct comparison of the hardware and software utilities follows from the constraint we impose on the marginal disutility toward hardware and software price, $\alpha^{p, s}=\frac{\alpha^{p, h}}{\psi}$. Consequently, the identification of $\psi$ originates from the differences in consumer responsiveness to hardware and software prices 12

\footnotetext{
${ }^{12}$ An equivalent alternative way of doing this would be to set the price coefficients identical in both markets, but allow the error variance in the software market to be multiplied by $\psi$, i.e. it would then be $\frac{\pi^{2} \psi}{6}$. In this approach, we would not need to multiply $W_{i t}^{c}$
} 
Next, we illustrate how the correlation between the value for hardware and software is identified. We detail the intuition behind the identification of the correlation parameter $\rho$ as follows. The key construct in this argument is the tying ratio defined as:

$$
T(t)=\frac{\text { Number of software titles sold in period } t}{\text { Installed base of consoles in period } t}
$$

Figure 4: Scenarios Representing Dynamics of Tying Ratio

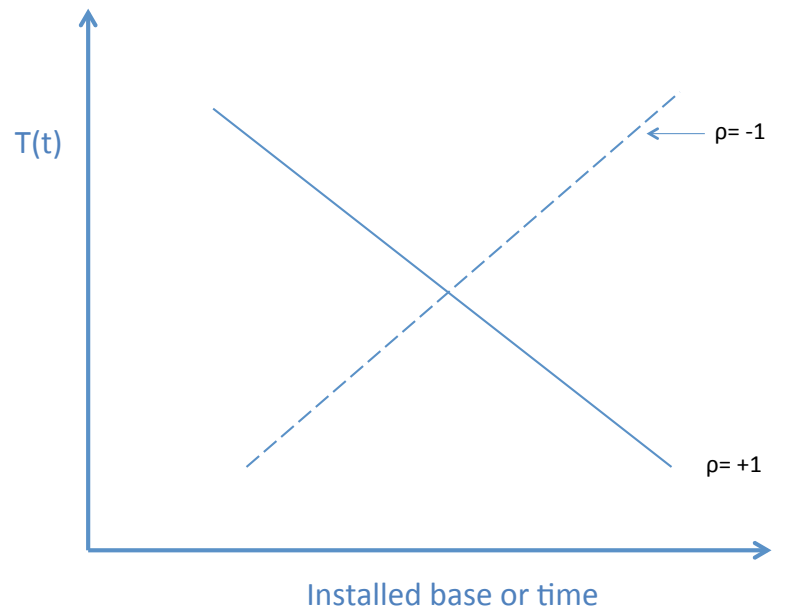

We use the variation in the tying ratio over time to provide the identification of $\rho$. Consider first the case of perfect negative correlation. For the purposes of illustration, we consider 4 segments of consumers: LL, LH, HL and $\mathrm{HH}$, where the first letter indicates the consumer's valuation for hardware, and the second indicates the consumer's valuation for software, e.g. an LH consumer would place a low value on hardware and high value on software. All else the same, a consumer who values software highly, i.e. LH or HH consumers will purchase more software titles in each period after they enter the software market compared with consumers who have low value for software, i.e. LL or HL consumers. Thus, the tying ratio changes depending on the entry of each of the consumer types over time. If $\rho=-1$, then we can have two types of consumers, LH or HL, but not LL or HH. On the other hand, if we have $\rho=+1$. then we have HH and LL consumers, but not LH and HL.

The tying ratio can either be upward sloping or downward sloping. Consumers value both hardware consoles and software games when they make a purchase decision in the hardware market, since the expected utility from software purchases is included in the utility of purchasing the console or bundle. Now consider two cases for the variation in tying ratio over time in Figure 4. When $\rho=-1$, i.e. the case with HL and LH consumers, consider the sequence of how these consumers enter the hardware market. If HL enters first followed later by LH, observe that by $\psi$ before adding it to the hardware utility formulation. 
the tying ratio increases over time, since consumers entering later value software highly and will purchase more software titles per period than consumers who enter earlier. On the other hand, if LH enters first followed by HL, then the tying ratio will decrease over time. Thus, the dynamic variation in the tying ratio gives us information about the sequence of entry of heterogeneous consumers with different valuation for hardware and software.

If $\rho=+1$, i.e. with $\mathrm{HH}$ and LL consumers, the only possible entry sequence is the following: HH enters first followed by LL, in which case the tying ratio is declining over time. Thus, if the tying ratio were increasing over time, we could infer that $\rho=-1$, whereas with a decreasing tying ratio, we could have two possibilities:

1. $\rho=+1$ and the sequence with HH consumers entering first, followed by LL consumers, or

2. $\rho=-1$ and the entry sequence with LH consumers entering first, followed by HL consumer.

To separate out these two possibilities, we leverage the response of console sales to price variation. We begin with the idea that HL or HH consumers who value consoles highly are less responsive to price variation in consoles than LL or LH consumers. Thus, if case (1) above holds, then the HH consumers who enter early are likely to be less sensitive to price than LL consumers who enter later, implying that the sales response to prices is less sensitive in early periods and becomes more responsive over time. However, if case (2) above holds, we must find that sales response to prices is more sensitive in early periods and becomes less responsive over time.

We find from the data that case (1) holds in our setting, consistent with our empirical finding that $\rho$ is positive. More generally, we note that we identify the correlation parameter $\rho$ through the combined variation in the tying ratio and the console sales response to price over time. Note that we have not used any arguments that require the presence of bundles in the data, and have suggested a rather general identification rationale for correlation in consumer preferences for two products that are sequentially purchased in a dynamic setting; the rationale relies on the purchase paths followed by consumers for the two products, i.e. console and videogames in our setting.

The determination of a potential market size for consoles is an important step in properly estimating console demand. One useful measure which is often used is the number of households with a TV in 2001, since the introduction of the GBA occurred in 2001. But the GBA is geared toward families with children so the measure of households with a TV seems to over estimate the potential market size 13 Therefore, we use the number of households who have children under 18 living at home as the appropriate market, approximately 35.7 million 14

The potential hardware market in period $t$ includes all consumers who have not made a purchase and remain in the market in that period. The construction of the potential market size reflects the idea that a consumer is a

\footnotetext{
13 Dube et al. 2010 and others use a similar approach.

${ }^{14} \mathrm{An}$ alternative approach would be to use an approach from Bass (1969) that illustrates how to infer the initial potential market size of a product from its sales data. "An approximation to the discrete-time version of the model implies an estimation equation in which current sales are related linearly to cumulative sales and (cumulative sales)" (Nair, 2007). Let $k_{t}$ and $K_{t}$ denote the aggregate sales of all consoles in month $\mathrm{t}$ and cumulative sales up to and including month $\mathrm{t}$, respectively. Also, let $k_{t}=a+b K_{t}+c K_{t}^{2}+v_{t}$ be the equation we estimate. Given the estimates, the Bass model implies the initial potential market size for all handheld consoles is $M=(a / f)$, where $\mathrm{f}$ is the positive root of the equation $f^{2}+f b+a c=0$ and a is from the regression above. We have tried using this model as an alternative to estimate the market size, and it gives a potential market size of 35.7 million.
} 
first time buyer and does not re-enter the market to purchase additional hardware. Again, it is important to note that while the discussion here provides intuition for the specific patterns in the data that move parameter values to vary in magnitude and sign, in practice all the variation in the data is used to estimate each of the parameters, given the intertemporal substitution possibilities available to consumers, and the linkage between the console and video game markets.

\section{Estimation}

We model heterogeneous consumers, with the consumer's type drawn from a discrete distribution of $N_{s}$ types. The coefficients are represented by $\alpha_{i}=\bar{\alpha}+\nu_{i} \Sigma^{\frac{1}{2}}$. We examine both Monte Carlo simulations in drawing individualspecific coefficients and then summing over the individuals to obtain a simulation-based approximation, as well as the quadrature approach Skrainka and Judd, 2011, which takes a direct polynomial approximation approach to compute the integral 15 Consistent with the dynamic demand literature, and that of studies of the video game industry, we set the discount factor to be $\beta=0.975$; however, recent studies have assumed a lower discount factor in these settings, and demonstrated that consumers discount such purchases by more than the rate implied by a "market interest rate."

Our dataset on the videogame industry spans the years 2001-2005. The Nintendo Gameboy Advance (GBA) introduced in Spring 2001 was a very significant leap in the handheld console market, and began a new generation of consoles. This feature of the data setting helps us in our estimation process because it mitigates concerns regarding whether the initial conditions present in the market at the beginning of our data may present persistent effects that may make accurate estimation of existing consumer inventory more challenging. Having data from the beginning of a significant market shift also permits us to consider a more tractable state space in the model, which enables our estimation process to converge in a reasonable time, and permits us to incorporate richer substitution effects and correlation between consumers' preferences for hardware and software.

We model the process of market evolution for the hardware and software markets jointly in a consistent manner that corresponds to aggregate choice behavior of individual consumers, who first purchase a console and then purchase software games. Given $N_{s}$ discrete types of consumers, we denote the sets of consumers of each type to be $\mathbf{S}_{\mathbf{1}}, \mathbf{S}_{\mathbf{2}}, \ldots, \mathbf{S}_{\mathbf{N}_{\mathbf{s}}}$. The potential market size for hardware in period $\tau$ can be captured by the variable $\mathbf{M}^{\mathrm{h}}(\tau)$, defined as a $\left(N_{s} \times 1\right)$-vector that captures the number of each of the $N_{s}$ types that have not purchased hardware by the beginning of period $\tau$ :

\footnotetext{
15 Specifically, we implement a Gaussian-Hermite quadrature approach with five nodes $\left(N_{s}=5\right)$ to obtain 25 segments from our two dimensions of heterogeneity.
} 


$$
\mathbf{M}^{\mathbf{h}}(\tau)=\left(M_{1}-\sum_{t=1}^{\tau-1} m_{1}(t), M_{2}-\sum_{t=1}^{\tau-1} m_{2}(t), \ldots, M_{N_{s}}-\sum_{t=1}^{\tau-1} m_{N_{s}}(t)\right)
$$

where $m_{s}(t)$ is the number of hardware purchases in period $t$ by consumers in segment $s$ and $M_{s}$ is the initial number of consumers of segment $s$ in period 0 . This formalizes the notion that each consumer who purchases a console or bundle exits the market for hardware. Consumers consequently enter the market for software video games, and the potential market size of video game purchasers in period $t$ includes consumers who have purchased consoles across all periods upto and including period $\tau$ :

$$
\mathbf{M}^{\mathbf{s}}(\tau)=\left(\sum_{t=1}^{\tau} m_{1}(t), \quad \sum_{t=1}^{\tau} m_{2}(t), \ldots, \quad \sum_{t=1}^{\tau} m_{N_{s}}(t)\right)
$$

Price endogeneity is known to be present for a variety of reasons. For instance, producers may set higher prices for games with higher quality, where the latter is not observable by the researcher, which will result in the price coefficients being biased upward. We also include game indicator variables, although with the use of fixed effects, the proportion of the unobservable product characteristic, which is not accounted for may still be correlated with price as a result of consumers and producers correctly observing and accounting for the deviation. Under this assumption, market specific markups will be influenced by the deviation and will bias the estimate of hardware or software price sensitivity.

In order to accurately estimate and identify a consumer's price sensitivity for consoles, software and bundles, we use instrumental variables to correct for endogeneity in prices. We use instruments which serve as proxies for marginal cost. The instruments for software video game titles include one-month lags of the Japanese to US exchange rate and the software producer price index. The producer price index is interacted with additional variables to capture differences between game characteristics, including age, rating and an indicator for integrated games. Specifically, the software producer price index is interacted with game rating, rating age as well as rating and an indicator variable for an integrated game. The implementation of such instruments captures and proxies for variable software costs among newer and older games, game type and quality levels.

Note that new products in most consumer electronics markets are introduced during the holiday season to take advantage of the higher expected demand. We account for this by using seasonality variables that pick up aggregate variation during the holiday period. Nevertheless, we acknowledge that strategic timing games played by the firm have the potential to result in endogeneity and possibly bias the results. We note that this is true in other markets as well (e.g. Berry et al. 1995 study the market for automobiles where new models are typically introduced in the summer, and Melnikov 2001 and Gowrisankaran and Rysman 2013 examine other consumer electronics markets where new products are introduced in the holiday season. 
Our estimation is based on GMM, and the criterion function gives us the estimator based on the orthogonality of the unobservable characteristics and the instruments for consoles, games and bundles ${ }^{16}$

$$
(\hat{\alpha}, \hat{\boldsymbol{\Sigma}})=\arg \min _{\alpha, \boldsymbol{\Sigma}}[\xi(\alpha, \Sigma) ; \quad \chi(\alpha, \Sigma) ; \quad \mu(\alpha, \Sigma)] \mathbf{Z} \mathbf{W} \mathbf{Z}^{\prime}\left[\begin{array}{c}
\xi(\alpha, \Sigma) \\
\chi(\alpha, \Sigma) \\
\mu(\alpha, \Sigma)
\end{array}\right]
$$

For hardware, we use a one month lag of the Japanese to US exchange rate again and the retail price of Nintendo's home video game console the GameCube as console price instruments. We also include an indicator variable of whether an additional console type is available (i.e. whether the Gameboy Advance SP has reached the market) to account for the multi-product pricing effect. The foreign exchange rate is a suitable instrument given the manufacturing of the console and games occur in Japan and would consequently affect the retail price of consoles in the US. We employ a one month lag of the exchange rate to allow for the duration between shipping, displaying and purchasing. Lastly, each instrument is interacted with a console indicator variable for either the GBA or the GBA SP to allow each variable to enter the production function of each console differently, similar to Villas-Boas 2007.

Handling the endogeneity of the bundle price is slightly more complicated than either software or consoles alone. One might think to only use the instruments from consoles to instrument for bundle price, but given our model specification where the unobservable bundle characteristic term would involve both hardware and software characteristics, this would still leave our bundle price correlated with the unobserved term . We correct for the endogeneity problem with the use of both software and console instruments. We report the first stage results for both software and hardware and an $F$-test for each set of excluded instruments in Table 4 , to demonstrate that the instruments are appropriate 17

We also supplement our estimation with the use of micro level survey data from Forrester Research's 2005 Technographics survey. This data allows us to form micromoments based upon the difference between the predicted distribution of consumers who own less/more than twenty video games to the actual distribution observed in the

${ }^{16}$ The matrix of instruments is constructed block-diagonally from the instruments for hardware, software and bundles and can be further specified as:

$$
\mathbf{Z}=\left[\begin{array}{cc}
Z^{h} & 0 \\
0 & Z^{s} \\
Z^{h} & Z^{s}
\end{array}\right]
$$

${ }^{17}$ First stage pricing model was jointly estimated given constraint from bundles. We employed the following model:

$$
\left[\begin{array}{c}
P_{g} \\
P_{c} \\
P_{b}
\end{array}\right]=\left[\begin{array}{cc}
x_{g} & 0 \\
0 & x_{c} \\
x_{g} & x_{c}
\end{array}\right] \times\left[\begin{array}{c}
\alpha_{g} \\
\alpha_{c}
\end{array}\right]+\left[\begin{array}{cc}
z_{g} & 0 \\
0 & z_{c} \\
z_{g} & z_{c}
\end{array}\right] \times\left[\begin{array}{c}
\beta_{g} \\
\beta_{c}
\end{array}\right]+\epsilon
$$

where $\mathbf{x}$ represent product characteristics and $\mathbf{z}$ the excluded instruments. 
Table 4: First Stage Results of Excluded Instruments

\begin{tabular}{lcc}
\hline Variable & Coefficient & Std. Error \\
\hline Software & & \\
Exchange Rate & $-0.0635^{* *}$ & 0.0101 \\
SoftwarePPI*Rating & $-0.0050^{* *}$ & 0.0015 \\
SoftwarePPI*Rating*Age & $0.0003^{* *}$ & $6.0428 \mathrm{e}-05$ \\
SoftwarePPI*Rating*Integrated & $-0.0134^{* *}$ & 0.0043 \\
SoftwarePPI*Rating*Integrated*Age & $0.0004^{* *}$ & $8.6356 \mathrm{e}-05$ \\
& & \\
Console & & \\
GameCubePrice*GBA & $0.1271^{* *}$ & 0.0169 \\
GameCubePrice*GBASP & $-0.5209^{* *}$ & 0.1045 \\
ExchangeRate*GBA & $-0.66644^{* *}$ & 0.1670 \\
ExchangeRate*GBASP & -0.0836 & 0.3964 \\
I[Additional Console] & $7.8191^{* *}$ & 1.6883 \\
\hline F-Statistic of Excluded Instruments & \multicolumn{2}{c}{39.9859}
\end{tabular}

data. We expand the traditional weighting matrix used in simulated GMM $\left(\mathbf{Z}^{\prime} \mathbf{Z}\right)$ along two elements in each dimension to include the inverse of the variance of the micromoments. For the variance, we use $\operatorname{var}_{m m}=\frac{p(1-p)}{n_{c}}$ where $p$ is the value of the moment in the data and $n_{c}=626$ is the number of consumers sampled in the survey. A low variance puts a significant weight on the micromoment and thus attempts to match it very closely. Moreover, these micromoments aid in the identification of the diminishing marginal utility associated with purchasing multiple video games over time, $\varphi\left(N_{g}\right)$. In practice we specify $\varphi\left(N_{g}\right)=\varphi \log \left(N_{g}\right)$. We also test for robustness with a linear functional form and the results do not differ qualitatively; we therefore only report the log specification below.

A potential concern in estimation of demand with a large choice set is the restrictive nature of the logit assumption with large and dynamic choice sets Ackerberg and Rysman 2005. It would be especially critical to control for the number of dimensions of unobserved product space in markets with large variety (like software with over 600 unique titles available in the market), and where both the actual products as well as the number of products undergoes a significant change from one period to the next. The challenge arises as a result of the way that standard discrete-choice models handle symmetric unobserved product differentiation. A realistic model of product differentiation ought to lead to more crowding when the number of products increases (since we might expect in our setting, some software titles to be more substitutable with others in a crowded field with hundreds of products). However, with the use of logit errors, the dimension of unobservable characteristics space expands proportionally to the number of products, since each product $j$ introduces its own "error" term, $\epsilon_{i j t}$ (Mariuzzo et al. 2010]). $\mathrm{A} \& \mathrm{R}$ demonstrate from microfoundations that the restrictive assumptions about the relationship between the number of products in a market and the dimensionality of unobserved characteristic space can lead to significantly biased estimates of elasticities and cross-elasticities, not to mention associated problems with the evaluation of counterfactual scenarios, and they develop a structural model that results in essentially the number of products $J_{t}$ appearing as an additive term in the consumer utility function. We include an appropriate transformation in the 
above consumer software model, and our derivation of the model from microfoundations would essentially replicate their logic.

\section{Results}

We detail our parameter estimates for the consumer's utility for both hardware and software utility in Table 5 below. We consider our model described above as well as a model with no consumer heterogeneity to serve as a comparison. We first focus on the software results and then proceed to the console and bundle results. The software market results conform to our expectations in magnitude and in sign. For instance, we determine that software utility declines at a decreasing rate as a game becomes older, as is evident from the corresponding signs on game age and age $^{2}$ variables. We also find significant consumer heterogeneity in valuation for software, $\sigma_{s}$. However, consumers do not have diminishing marginal utility towards holding numerous video games.

Table 5: Estimation Results

\begin{tabular}{|c|c|c|c|c|}
\hline \multirow[b]{2}{*}{ Variable } & \multicolumn{2}{|c|}{$\begin{array}{c}\text { No Consumer Heterogeneity } \\
\text { Dynamic Model }\end{array}$} & \multicolumn{2}{|c|}{$\begin{array}{l}\text { With Heterogeneity } \\
\text { Dynamic Model }\end{array}$} \\
\hline & Coefficient & Std. Error & Coefficient & Std. Error \\
\hline \multicolumn{5}{|l|}{ Software Utility Parameters } \\
\hline Software Constant $\left(\bar{\alpha}^{s}\right)$ & $0.6680^{* *}$ & 0.0034 & $0.3558 * *$ & 0.0029 \\
\hline Game Age & $-0.0043^{* *}$ & 0.00015 & $-0.0034^{* *}$ & 0.0001 \\
\hline Game Age ${ }^{2}$ & $0.00003^{* *}$ & 0.000003 & $0.00003^{* *}$ & 0.000003 \\
\hline $\log \left(\left|\mathbf{S}_{\mathbf{t}}\right|\right)$ & $-0.03184^{* *}$ & 0.00119 & $-0.0253^{* *}$ & 0.00101 \\
\hline Scale Parameter $(\psi)$ & $0.8336^{* *}$ & 0.1241 & $0.9752^{* *}$ & 0.1502 \\
\hline Diminishing Marginal Utility $(\varphi)$ & 0.0012 & 0.5228 & 0.0014 & 0.1840 \\
\hline Sigma Software $\left(\sigma_{s}\right)$ & & & $0.7550^{* *}$ & 0.3436 \\
\hline \multicolumn{5}{|l|}{ Console Utility Parameters } \\
\hline Hardware Constant $\left(\bar{\alpha}^{h}\right)$ & $-0.5175^{* *}$ & 0.0053 & $-0.4261^{* *}$ & 0.0076 \\
\hline Bundle Constant $\left(\alpha^{b}\right)$ & $-0.0743^{* *}$ & 0.1010 & $-0.0749^{* *}$ & 0.0012 \\
\hline Price & $-0.0561^{* *}$ & 0.0152 & $-0.0649^{* *}$ & 0.0138 \\
\hline Age of Console & 0.0011 & 0.000475 & $0.0061^{* *}$ & 0.00058 \\
\hline Age of Console ${ }^{2}$ & $-0.00007^{* *}$ & 0.00001 & $-0.00006^{* *}$ & 0.000015 \\
\hline Sigma Console $\left(\sigma_{h}\right)$ & & & $4.2699^{* *}$ & 0.3883 \\
\hline Correlation $(\rho)$ & & & $0.7111^{* *}$ & 0.2514 \\
\hline GMM Objective & 392.4387 & & 12.0435 & \\
\hline \multicolumn{5}{|c|}{ Game, Console and Month of Year FE in all models not reported } \\
\hline
\end{tabular}

We now discuss the consumer preference parameters for the hardware market, consisting of consoles and bundles. As we discussed above, we assume a consumer has the same marginal disutility towards the price of a standalone console or a bundle, i.e. the price coefficients for bundle price and console price are identical. Yet software price sensitivity is linked to hardware sensitivity by the scaling factor $\alpha^{p, s}=\frac{\alpha^{p, h}}{\psi}$, where the marginal disutility to 
software price is equal to a scaled value of the disutility of hardware price. We determine the scale coefficient $\psi$ to be statistically significant. We find the hardware price coefficient $\alpha^{p, h}$ to be negative and significant as expected, so that consumers have a marginal disutility toward higher price. Recall that we introduce heterogeneity in a consumer's preference for hardware, i.e. $\alpha_{i}^{h}$ is individual-specific. We find that there is a significant degree of consumer heterogeneity toward consoles $\left(\sigma_{h}\right)$ and that consumer preferences for games and consoles are highly positively correlated, and both heterogeneity and correlation are significant. Heterogeneity plays a vital role in the model as consumer valuation for hardware and software dynamically alters the distribution of consumers who own a console, and who are therefore present in the software market.

Figure 5: Distribution of (a Sample of) Consumer Types Over Time for the Console Market

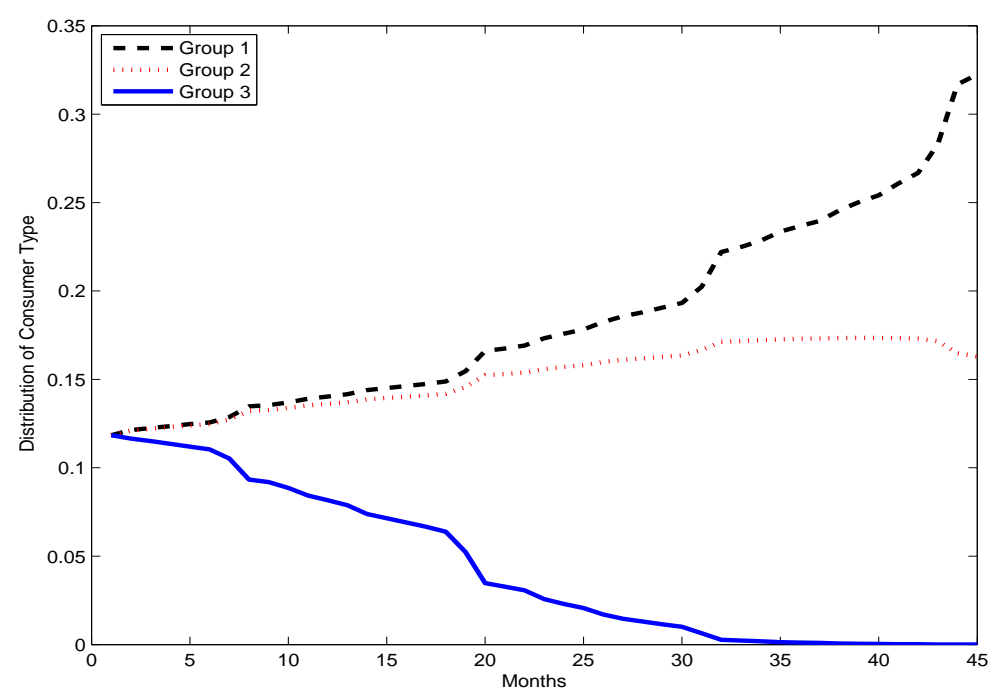

Figure 5 reflects the distribution of three consumer types over time for the hardware market. In estimation, we discretize consumers into $N_{s}=5 \times 5=25$ different segments or groups, based on their value for hardware and software. The fraction of consumers belonging to each specific segment changes dynamically in each market, depending on consumers' purchase decisions. Group 1, for instance, is a group which possesses a relatively low preference for hardware and software. With this low preference consumers within this group postpone consumption which causes its percentage of consumers who remain in the market for consoles to increase over time (similarly for Group 2 for the first part of the data period). On the other hand, Group 3 has relatively large preference for hardware and software and as a result do not postpone consumption into the future as much as groups 1 and 2; thus, these consumers leave the hardware market earlier, and their numbers subsequently decline over time. Lastly, we examine whether consumers value bundles over and above the individual products in the bundle (console \& game). We find differences between bundles and consoles. Consumers have a different seasonality effect for bundles than for consoles.

We next investigate consumer substitution patterns, and Table 6 provides the own and cross price console and 
Table 6: Console and Bundle Elasticities

\begin{tabular}{lccccc}
\hline & GBA & GBAMA2 & GBAMK & SP & SPMA4 \\
\hline Gameboy Advance (GBA) & 1.09 & -0.05 & -0.14 & -0.48 & -0.01 \\
GBA with MarioAdvance2 (GBAMA2) & -3.82 & 4.09 & -0.05 & -0.01 & -0.001 \\
GBA with MarioKart (GBAMK) & -4.76 & -0.003 & 9.03 & -0.01 & -0.001 \\
GBA SP (SP) & -0.79 & -0.01 & -0.01 & 1.11 & -0.06 \\
GBA SP with Mario Adv4 (SPMA4) & -1.24 & -0.02 & -0.01 & -4.13 & 6.01 \\
\hline
\end{tabular}

Note: Cell $i, j$, where $i$ indexes row and $j$ column, gives the precent change in the total quantity of row $i$ with a one percent change in price of column $j$

bundle elasticities estimates. We evaluate the elasticity by considering a $1 \%$ price cut that is made permanently, and is known by consumers as well as the firm to be a permanent cut, i.e. there is no uncertainty regarding the future price cut unlike the case of a limited-time promotion. The model predicts that a permanent $1 \%$ percent reduction in the price of a console would lead to an approximately 1-9\% increase in the total number of a given console sold during the time period. Also note that the own price elasticity for bundles is substantially larger than for consoles. This result is consistent with previous literature where consumers are more price sensitive to bundles than components Sharpe and Staelin, 2010]. The cross-price elasticities correspond to the effect of a price cut on consoles on sales of bundles, and vice versa and range from 0 to $-5 \%$. Moreover, the off-diagonal elements are negative and the estimated cross-price elasticity measures are consistent with the belief that a standalone console's closest "competitor" is the bundle with the particular console included. For instance, the closest "competitor" to the standalone Gameboy Advance console is the GBA bundle with Mario Advance 2 and not the GBA SP; such a result would not have been apparent without modeling the microfoundations of consumer demand.

Figure 6 displays the intertemporal handheld console market elasticities. We present three variants of an elasticity measure to highlight the role of consumer expectations, as is typical in these settings. We compare the effects of a temporary $1 \%$ price decline at time period $t$ when consumers believe the price change is temporary to one in which consumers believe it is permanent. Our last measure we include is a $1 \%$ price decline that is permanent and is believed to be permanent. In all cases, price changes are not expected in advance by the consumer.

The elasticity measures correspond to the median time period in the data, i.e. month 22 . We find that expectations play a vital role in how consumers respond to price changes. For a temporary and unexpected $1 \%$ price change, we see that sales remain unchanged prior to period $t$ and increase by roughly several percentage points in that period. We also find that such an unexpected and temporary price change only results in a gain in sales in the period the price decline is present and a subsequent decline in sales, suggesting inter-temporal substitution by consumers. However, when the temporary price change is believed to be permanent, we see a smaller reaction in consumer sales than when the price decline is temporary and believed to be temporary. This is due to consumers' beliefs that such a price decline in period $t$ will be available next period and so they postpone their purchase until a future period. But when period $(t+1)$ prices return to their higher price levels consumers who otherwise would 
Figure 6: Industry Dynamic Price Elasticities

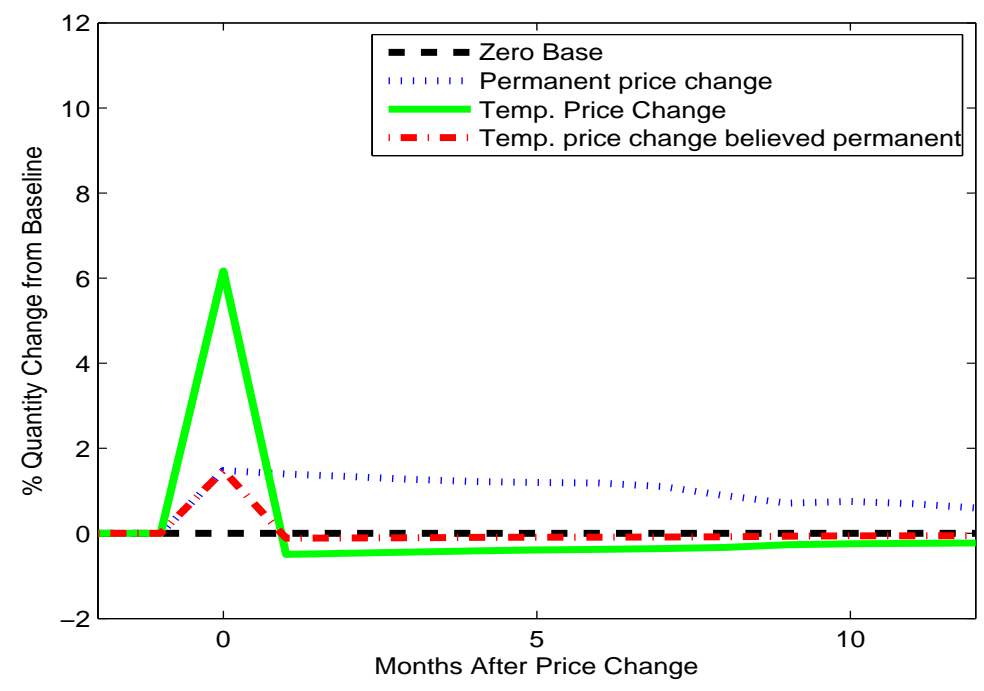

have purchased in period $(t+1)$ with lower price do not. What is evident from this figure is that temporary price cuts only have temporary effects on sales, the positive increase in sales is only temporary. Lastly, we analyze price elasticities for video games, and find that all games have a positive price elasticity ranging from 0.25 to 2.0.

We detail the results from several other model specifications, as well as the model fit results in Appendix A. The other model specifications check for robustness of the primary effects with and without myopic consumers in both hardware and software markets, and including forward-looking "gift-giving" consumers who only enter in the holiday season, and evaluate the results when the bundle seasonality is built up from the combined seasonality effects from hardware and software. We find the results to be qualitatively similar to the results in Table 5

\section{Counterfactuals}

A key objective in developing our structural model is to enable us to evaluate the effect of realistic counterfactuals, some of which may involve factors that are outside the range of the data. More specifically, these counterfactuals help us answer the questions listed in $\S 1$. Since our goal is to understand the effectiveness of bundling as a product strategy, we compare market outcomes with those that result when bundling is eliminated as an option for the firm. This baseline scenario will allow us to evaluate the degree to which bundles may potentially cannibalize sales of standalone consoles and how they may induce consumers to advance purchases.

It is important to note that proceeding with the same price trajectory (as in the data) under counterfactual situations would lead to a somewhat mechanical result since deleting an option (i.e. the bundle) from consumers' hardware choice sets forces overall demand for hardware to fall by making it essentially impossible to have everyone substitute to one of the inside goods, i.e. some consumers will certainly choose the outside option in such a case. This counterfactual would lead to the erroneous conclusion that the observed strategy of offering a bundle is better 
because the firm would not alter its price levels when the bundle option is removed 18 We need to recognize that when the product strategy is altered in the counterfactuals (e.g. the case with no bundles), firms would expect to set different prices than they currently do. In addition, there are other market-specific constructs that remain unchanged in the counterfactual setting, e.g. no entry or exit of firms. Although modeling the supply-side role of setting prices dynamically is unrealistic given the complexity of our setting, and the consequent tractability issues that arise, we attempt to alleviate this limitation by two different and complementary approaches.

First, we approximate the pricing decision of the firm by an $A R(1)$ process, as is common in the marketing literature on dynamic structural models, with the following specification:

$$
P_{j t}=\alpha_{1}+\alpha_{2} P_{j t-1}+\alpha_{3} \mathbf{I}(\text { Bundle })+\mathbf{X}_{\mathbf{j t}} \beta+\varepsilon_{j t}
$$

to understand how the monopolist might set prices depending on both product characteristics, past prices and the presence or absence of bundles. We employ a methodology from the treatment effects literature to measure the average price effect bundles have on standalone console prices. Second, we allow standalone console prices to vary by $-10 \%$ to $+10 \%$ from the observed price data to allow the firm additional price flexibility that might not be completely captured in the above specification in equation (29). We implement each of these price adjustments as robustness checks to understand how sales would change if the firm were to try a variety of different pricing strategies. While such a treatment is not equivalent to a full-equilibrium dynamic pricing equilibrium model, we condut sensitivity analysis of assumed price changes to ensure our conclusions hold across a reasonable range of price levels.

\section{Counterfactual 1 (No Bundling)}

We begin with the alternative scenario of eliminating mixed bundling as a product strategy. The console price regression result shows that when bundles are not present, console prices are higher than compared to the case when bundles are present. In the counterfactual, we appropriately adjust standalone console prices upward in periods when bundles were available. The results are detailed in Table 7 and the dynamics of the change in installed base corresponding to the counterfactual are illustrated in Figure 7. We find that the use of mixed bundling leads to cannibalization of over half a million consoles, but the effect is more than offset by the sale of bundles, leading to an overall increase in hardware unit sales. Total hardware sales are higher by approximately one-hundred thousand units under mixed bundling, compared with a "no bundling" setting. While it is useful to note that bundling increases hardware sales, it is even more important to understand its effect on software sales.

As Table 7 details, even a small increase in hardware sales generates over millions more videos games sold with a significant fraction being high margin integrated games. Overall revenues form hardware, software as well as

\footnotetext{
${ }^{18}$ We thank a reviewer for suggestions regarding the pricing supply-side role.
} 
Table 7: Counterfactual 1: Eliminate Bundles

\begin{tabular}{lllll}
\hline & Data & Model (Base) & CF (No Bundling) & Model-CF \\
\hline Console Sales & $25,891,953$ & $25,976,646$ & $26,441,379$ & $-464,733$ \\
Bundle Sales & 563,678 & 563,886 & 0 & 563,886 \\
Independent Software Sales & $80,845,492$ & $83,270,691$ & $78,088,240$ & $5,182,451$ \\
Integrated Software Sales & $31,400,467$ & $32,308,682$ & $30,311,074$ & $1,997,608$ \\
Discounted Revenue & $2,112,371,134$ & $2,138,564,056$ & $2,087,724,081$ & $50,839,975$ \\
\hline
\end{tabular}

overall revenues from mixed bundling are higher than with a component only product strategy 19

We see that bundling is particularly successful in time-shifting purchases of consoles earlier in the life cycle. Figure 7 depicts the important dynamics of how the hardware installed base under a console only strategy differs from a mixed bundling strategy. Examining Figure $7 \mathrm{~b}$, we find that the consumers who have a low value for hardware and both low and high value for software are the most impacted by bundling, and accelerate their purchases when bundles are present in their choice sets. We also find that consumers who have a high value for hardware are not impacted by the presence of bundling. These results overall suggest that bundling has the potential to attract lower-valuation segments of consumers to purchase when they might otherwise not do so. It thus provides an additional mechanism to dynamically segment different types of consumers, and induce substitution from the "no purchase' or 'delay purchase' options. It is noteworthy that high type consumers do not substitute away from component products to bundles, since bundles are lower in valuation and priced lower than the sum of component products.

Although the results illustrate an increase in both hardware and software sales from a mixed bundling strategy, the timing of these additional adoptions is important. A console manufacturer would prefer to sell as much hardware as early in its life cycle as possible, which results in an increase in software demand due to indirect network effects. We note that the installed base only considers the benefits of bundling on the hardware market. Thus, if software producers develop more software when there is a larger installed base, the true effects of bundling are likely to be even higher than we have documented.

\footnotetext{
${ }^{19}$ Note that revenues are calculated by summing over three monthly revenues streams with 45 months of data: (i) hardware sales (bundles and consoles) (ii) royalty fee from independent software sales, which is set at $\$ 8$ per software title sold, and (iii) revenue from integrated games, i.e. those produced by the monopolist firm. Once monthly revenues are calculated, we discount revenues back to the first period of the data.
} 
Figure 7: Counterfactual 1: Dynamics of Installed Base

(a) Difference in Installed Base with and without

Bundling

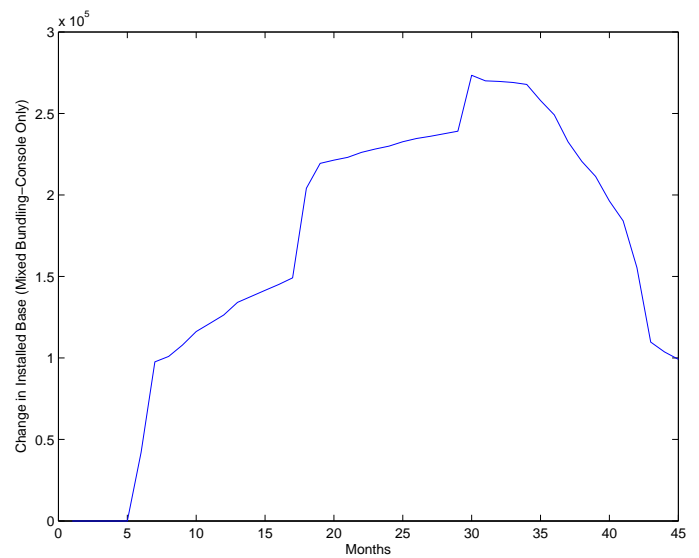

(b) Consumer Heterogeneity and Bundling Effectiveness

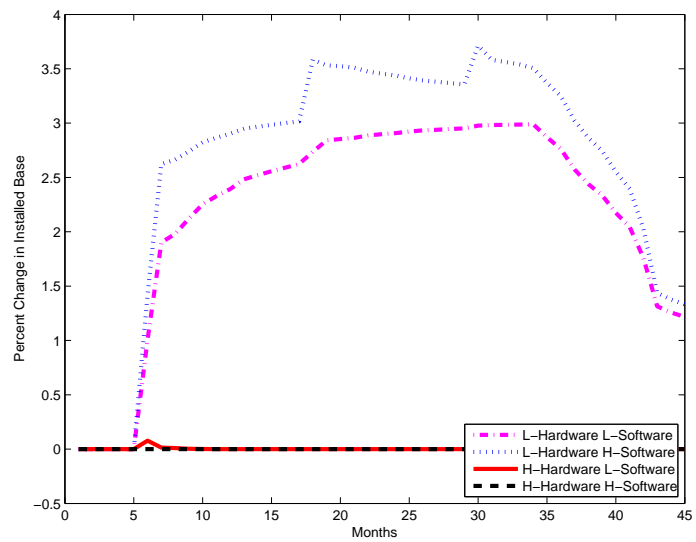

\section{Counterfactual 2 (Pure Bundling)}

Next, we evaluate the scenario with pure bundling, where consumers must buy a bundle in order to own a console, i.e. standalone consoles are not available as an option for consumers. Intuitively, pure bundling can be more profitable when consumers who value either of the component products switch to purchasing the bundle, which can be positioned to extract higher surplus from consumers, leading to more revenue for the firm. On the other hand, if consumers switch more to the outside option in the absence of component products, then pure bundling could result in revenue losses. We note that the literature reviewed in $\$ 1$ finds support for mixed bundling being more profitable than pure bundling, as well as the reverse case. We evaluate which effect dominates in our setting, i.e. do pure bundles increase or decrease revenues? Note that since bundles are not present in every period in the data, we allow the standalone console to be sold in those periods.

Figure 8 provides the results of the difference between pure and mixed bundling, and we find that mixed bundling dominates pure bundling with respect to a revenue measure. Given that we do not observe what the bundle price would be without the pure console, we run our counterfactual simulation for a range of prices, from $10 \%$ lower to $10 \%$ higher than the bundle price observed in the data. We find that there are highly significant revenue losses from pure bundling compared with mixed bundling, with differences being at least $35 \%$.

From the first two counterfactuals, we determine that mixed bundling dominates pure components as well as pure bundling. Our conclusion is based on the role played by dynamics and intertemporal substitution. The firm can attract consumers who might have only purchased a console in later periods by offering a bundle, which leads those consumers to purchase bundles earlier. For such a consumer, the bundle is a more compelling value proposition 
Figure 8: Counterfactual 2: Pure Bundling ( $\frac{\text { Pure Bundling-Mixed Bundling }}{\text { Mixed Bundling }}$ )

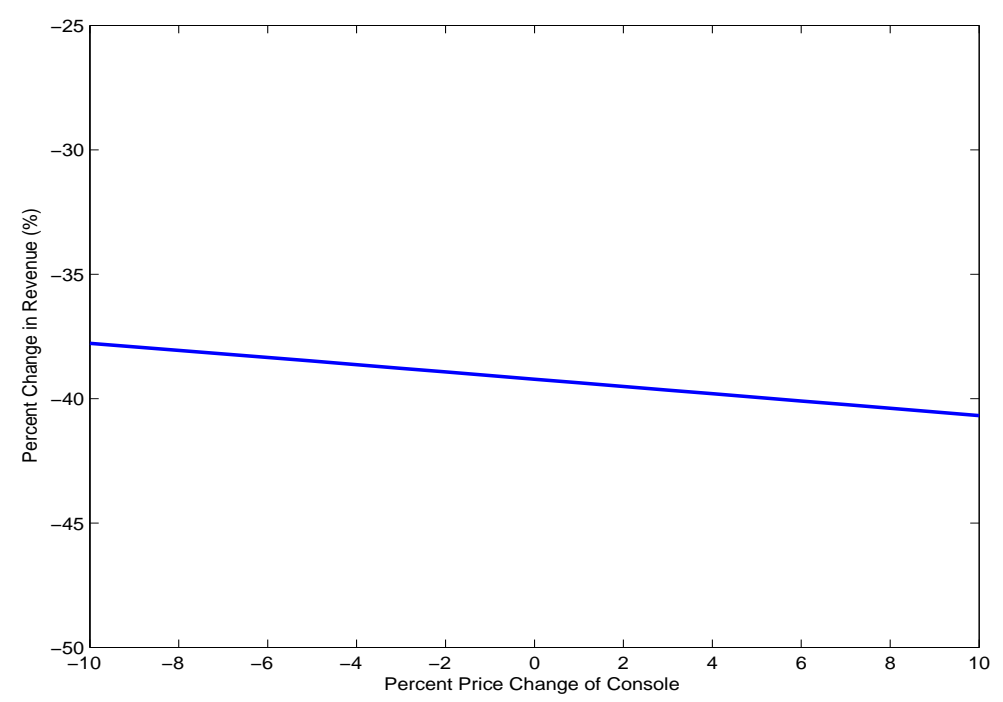

than the console, and in its absence, they might wait for the console price to decline before making a purchase. Consequently, by pulling hardware sales forward, the firm is able to get these consumers to the software market earlier, leading them to purchase more software over time, which in turn leads to better extraction of consumer surplus over the product life cycle.

Overall, we demonstrate an additional driver of bundling effectiveness, i.e. dynamic consumer segmentation by which firms can target specific segments of consumers by including bundles in the consumers choice set. We next investigate the role played by correlation, heterogeneity and the indirect network effect in the relative profitability of mixed bundling over a pure component product offering.

\section{Counterfactual 3 (Correlation and Heterogeneity)}

A primary explanation in the literature has been that a higher degree of negative correlation is more helpful for firms to extract surplus through bundling, since consumers become more homogeneous in their valuation for the bundle when correlation is negative McAfee et al., 1989, Schmalensee, 1984a. The estimation results from our model point to the finding that consumer value for hardware and software are highly positively correlated, consistent with the notion that consumers value the overall experience from gaming, which requires them to purchase both hardware consoles and software games. We find that in our dynamic setting, mixed bundling can be more profitable than pure components even with a high and significant positive correlation.

From Figure 9, we find that bundling can be profitable even when consumer valuations are highly positively correlated. In this figure, we allow the correlation parameter to vary between -1 and +1 . We also allow the change in the standalone console prices to vary within a range of $-10 \%$ to $+10 \%$ when bundling is eliminated to show 
Figure 9: Counterfactual 3a: Correlation $\rho\left(\frac{\text { Mixed Bundling-Pure Components }}{\text { Pure Components }}\right)$

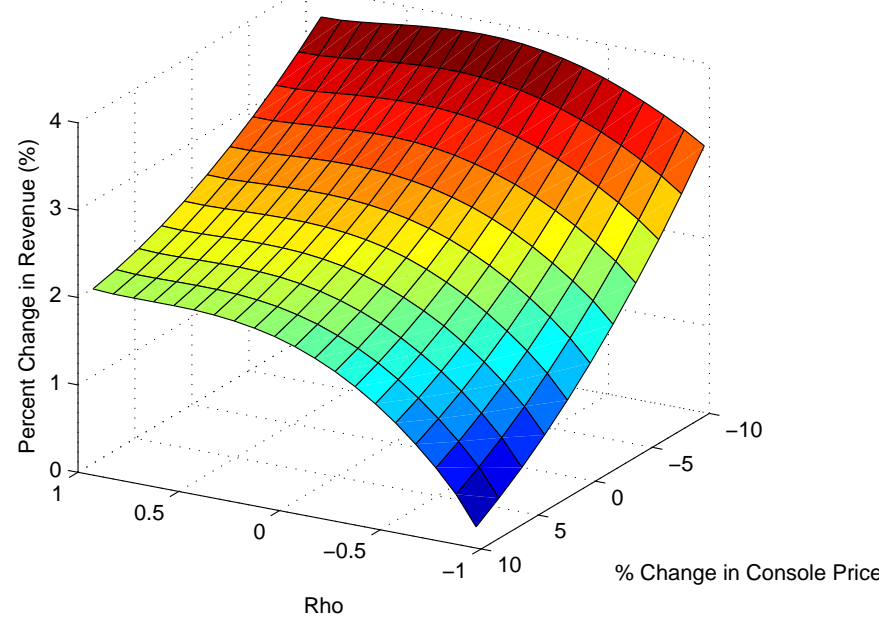

robustness of the effect for a range of price changes ${ }^{20}$ Consistent with the theoretical literature we find that correlation of preferences plays a significant role in the effectiveness of bundling, but to the best of our knowledge, this effect has not been empirically recognized and quantified. One challenge in doing so might be to identify correlation in aggregate data, and we provide a novel identification strategy to obtain this finding.

Another point of note is that the dynamic consumer segmentation works best in cases of high positive correlation, whereas the classic homogenization effect works best when consumer valuations are negatively correlated Schmalensee, 1984b]. Consider the case of perfect positive correlation to see why this would happen. With $\rho=+1$, consumers are either HH or LL (i.e. high valuation for both software and hardware, or low for both). In such a case, the LL type consumers would delay purchasing the product until hardware prices fall and there are sufficient software titles available in the marketplace. These are exactly the consumers who can be induced by the firm to purchase earlier by introducing a low priced bundle in the product line. On the other hand, when consumer valuations are perfectly negatively correlated, i.e. $\rho=-1$, we have LH or HL type consumers only and these consumers value have an intermediate value for bundle, making it more difficult to induce them to purchase earlier. Thus, the mechanism of dynamic consumer segmentation we investigate in this paper can be effective under conditions when the classic homogenization effect is not strong (or even present).

We next examine the role of consumer heterogeneity, and show that greater heterogeneity increases the ability of firms to extract greater surplus from consumers. In Figure 10, we present the results of a simulation where we eliminate mixed bundling, but allow the degree of consumer heterogeneity to vary. Specifically, we allow the recovered consumer heterogeneity values for software and hardware to vary from $\frac{1}{2}$ to $1 \frac{1}{2}$ times their estimated values, and we also allow component console prices to vary by $\pm 10 \%$ compared with a pure component regime.

\footnotetext{
${ }^{20}$ Chao and Derdenger 2013 determine from a theoretical model of mixed bundling in a two-sided market framework that standalone console prices rise under a pure component regime relative to a mixed bundling regime.
} 
Figure 10: Counterfactual 3b: Sigma ( $\left.\frac{\text { Mixed Bundling-Pure Components }}{\text { Pure Components }}\right)$

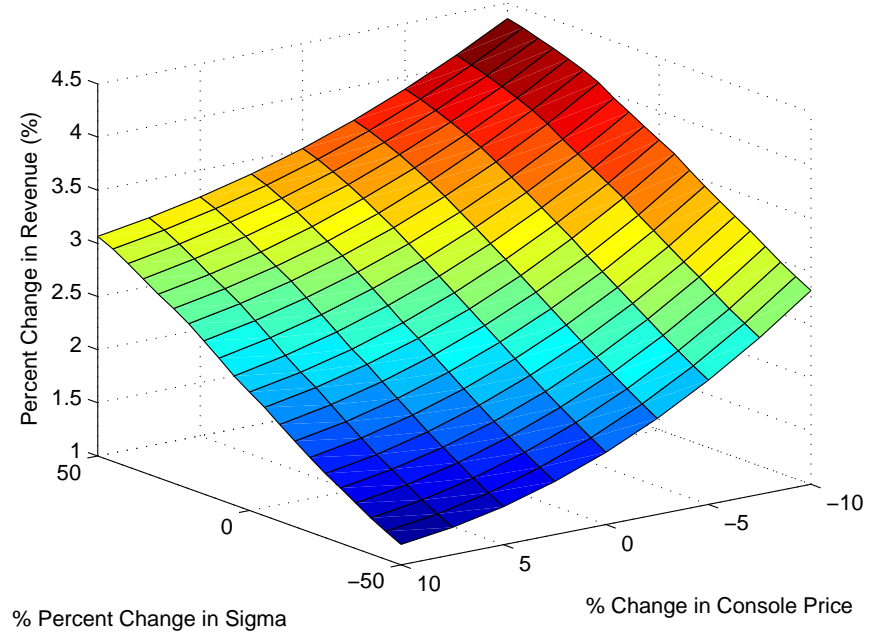

We observe that as consumer heterogeneity increases, the value of mixed bundling also increases. Such an increase in profitability is a direct result of the ability of the bundle to pull consumer sales forward, particularly for those consumers who do not separately value hardware enough to purchase it on its own early in the life cycle; this effect in turn leads to more software sales over time. In summary, with a lower degree of heterogeneity among consumers, the ability to pull sales to earlier periods through the introduction of a bundle is reduced, resulting in a smaller relative impact of mixed bundling. Taken to the extreme, when all consumers are identical, the dynamic consumer segmentation effect is highly diminished, and we find that this effect played a significant role in ensuring the effectiveness of bundling.

\section{Counterfactual 4 (Indirect Network Effect)}

Next we examine Counterfactual 4, where we investigate whether an increase in the indirect network effect makes bundling more or less effective for the firm. We set the marginal benefit of each game for the consumer to increase by altering the software utility, as included in the hardware utility model. We use a range of one to two times the size of this effect as recovered from the data, which allows us to determine dynamic comparative statics of the network effect and mixed bundling. We again allow console prices to vary by $\pm 10 \%$ from of the observed console price. Consumers attach a higher or lower utility to the present discounted utility of software game titles when considering a hardware purchase in this counterfactual scenario. In Figure 11, we find that a higher network effect decreases the relative effectiveness of mixed bundling, but does lead to a significant increase in overall discounted revenues. Moreover, we find that bundling has a smaller effect when the indirect network effect is stronger, suggesting that its use as a product strategy is likely to be less effective in settings with strong interdependencies between hardware and software. Thus, we find that bundling could serve as a substitute to network effects rather than as a complement, 
and expect bundling to be more effectively used in cases where the firm is not able to create a product with strong network effects. However, there are regions where we allow for significant decline in price changes from the observed data, where bundling serves as a slight complement to strong network effects. Thus, overall, we would expect the interaction between bundling and network effects to be more nuanced.

Figure 11: Counterfactual 4: Network Effects ( $\left.\frac{\text { Mixed Bundling-Pure Components }}{\text { Pure Components }}\right)$

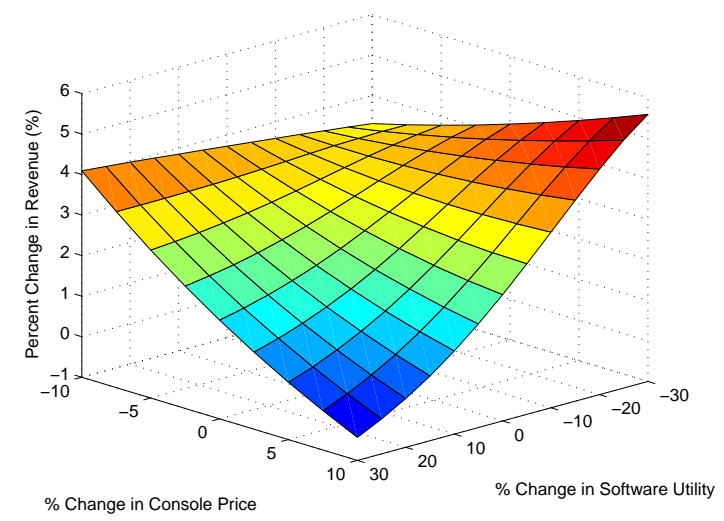

As stated above, we make several simplifying assumptions in order to tractably analyze the impact of bundling. Note that we do not model the software firms are unable to reoptimize their entry and exit decisions for video games across counterfactuals. Although these assumptions create obvious limitations, we expect that the qualitative findings would persist in equilibrium, since they are data-driven and persist across a variety of model specifications.

Let us first begin with the assumption of holding stand alone software prices constant. If we follow a similar method to analyzing the impact of bundling on component console price as in counterfactual one, we would determine that component prices of bundled software when bundles are not present would decrease by a very small amount. Moreover, given that these video games are bundled late in their life cycle, the increase in pure component revenue relative to revenue when bundle software prices do not change would be small, leading to the conclusion that the bias associated with our assumption of holding software prices fixed across regimes is rather small. Next, we assume that software firms do not reoptimize the entry and exit of video games when we simulate an alternate regime. Again, this assumption should not qualitatively change our results given that the number of consumers who purchase hardware in a component only simulation declines while holding video games constant to the number and utility observed in a mixed bundle setting. Thus, if we were to allow software firms to reoptimize their entry and exit decisions for each game, we would expect that the number of games would decline, since entry and exit is a function of the number of consumers holding hardware consoles. Consequently, this decrease would lead to an even smaller number of consumers purchasing hardware, lesser software titles being sold and less total discounted revenue. 


\section{Discussion, Limitations and Conclusion}

We have examined the impact of bundling decisions of firms on consumer choices and market outcomes in a setting with complementary goods, using data from the handheld video game market consisting of hardware consoles and software games. Such a setting involves consumer purchases of durable goods that are characterized by indirect network effects, and where dynamics are especially important. We develop a dynamic model based on individual consumer behavior, where consumers face a choice of consoles, bundles and video games over time. Consumers first enter the market for hardware (consoles), and following a hardware purchase, they enter the software (video games) market. The model allows for forward-looking consumers who have expectations over the future evolution of hardware and video games, and captures this effect in a tractable manner, building upon recent work on dynamic demand models. We set out to examine how bundling as a product strategy affects sales of consoles (cannibalization) as well as video games, how bundling creates value through the homogenization effect, and how the presence of indirect network effects interacts with bundling.

We evaluate the mechanisms through which bundling complementary products creates value for consumers, over and above the valuation for bundle components. . We find that consumer valuation for component products, i.e. hardware and software, are positively correlated across the population of consumers. We identify a new driver of bundling effectiveness, resulting form dynamic consumer segmentation: bundles attract some segments of consumers to advance their purchases, and others to enter the market when they might not have otherwise. Note that this is a mechanism that operates independent of the classic "bundling reduces consumer heterogeneity in valuation" mechanism. We find that the dynamic consumer segmentation explanation allows bundling to be even mroe effective with positive correlation in consumer valuations for the two products because it has the power to induce those consumers who have low valuations for both products to intertemporally substitute purchase to enter the market earlier. On the other hand, the classic homogenization mechanism is likely to be of limited value, since consumer valuations for component products are positively correlated. The mechanism of dynamic consumer segmentation underlying the effectiveness of bundling is likely to be especially important in markets where consumers have heterogeneous valuations for products, and where intertemporal tradeoffs are especially important, e.g. durable goods or technology products.

We also evaluate whether alternative strategies like pure components or pure bundling may perform more effectively in place of mixed bundling. We find that console sales diminish in the absence of mixed bundling, and that consumers who had purchased bundles may not always purchase pure consoles, even though consoles may be cheaper than bundles. Video game sales drop by millions of units and the overall discounted revenue reduces by more than fifty million dollars. We find that bundling serves as a substitute to network effects, suggesting it can be better leveraged when such effects are weaker, and that its relative benefit might not be as much in markets with strong network or winner-take-all effects. Overall, we find bundling to be a flexible product strategy option, 
which allows firms to create entire product lines where only one product existed. While our findings on bundling are especially true for products with low or zero marginal costs, its effectiveness as a product strategy would be interesting to examine in other markets.

The study could be enhanced by further work that could address the limitations arising from the nature of the data and from the viewpoint of computational tractability. First, we develop a model based on individual consumers with forward-looking behavior, but only have access to aggregate market-level aggregate data. While this limitation is shared by most empirical studies of durable goods, having access to dis-aggregate individual-level data would enable us to better examine the heterogeneity in consumer behavior, and could lead to more precise marketing suggestions. However, we note that to characterize how bundling results in homogenization of valuations requires aggregate type data, or at least a large sample individual data from a representative set of consumers sampled across the heterogeneity spectrum.

Second, we do not model the firm's decision to introduce products at specific time periods (often in the holiday season as in most consumer electronics markets), which has the potential to bias our findings due to endogeneity constraints. Although we do not model firm decisions as in most dynamic demand models, we instrument for endogenous pricing and evaluate our findings across a range of counterfactual price levels, and find our results to hold. Third, from a modeling perspective, we do not allow individual households to purchase hardware in a repeated manner (they would be treated as different households if they made a repeat hardware purchase). Fourth, we do not fully model the consumer problem of dynamically choosing the optimal software library or portfolio of titles, since that would be intractable. Rather, we aim to capture the dynamics in the software market by modeling decreasing marginal utility for software as well as capturing the dynamic evolution of the software market through the evolving inclusive value. Fifth, our results may be biased due to limited bundle availability. Suppose that some consumers did not have bundles in their choice set. Our model assumes all consumers have bundles in their choice sets when bundles are available, and we find that many consumers choose not to buy bundles when offered the opportunity. If instead there were limited availability, many consumers would not have bundles in their choice set, and for consumers who had bundles in their choice set, bundles would receive a higher probability of purchase, implying they have a higher utility. This would lead us to expect the bundle fixed effect to be less negative that it currently is in the results. Thus, it has the potential to strengthen the purchase acceleration or dynamic consumer segmentation, i.e. the mechanism underlying how the firm obtains higher revenue from mixed bundling.

While our results lay the ground for a more empirically grounded understanding of bundling, there remain several interesting avenues for further research. It would be useful to examine the dynamic introduction and phase-outs of bundles, including the choice of games to be included in bundles and the choice of whether exclusive software should be marketed only as part of bundles. Another prospect is to evaluate the competitive dynamics between firms in introducing bundles, and in characterizing the incentives of third-party game producers to sell 
their products bundled with consoles. More broadly, our examination of the role for bundling brings up the issue of how firms ought to think about product line decisions for individual products when bundling allows them more flexibility in enabling the creation of differentiated products that can be designed to be temporary or persist for long periods. Bundling thus can be an effective, practical and profitable component of a firm's product strategy.

\section{References}

D. A. Ackerberg and M. Rysman. Unobserved product differentiation in discrete-choice models: Estimating price elasticities and welfare effects. RAND Journal of Economics, 36(4):771-788, 2005.

W. J Adams and J. L Yellen. Commodity bundling and the burden of monopoly. The Quarterly Journal of Economics, 90(3):475-498, 1976.

A. Amelio and B. Jullien. Tying and freebies in two-sided markets. IDEI Working Papers, 2007.

M. Armstrong and J. Vickers. Competitive non-linear pricing and bundling. Review of Economic Studies, 77(1): $30-60,2010$.

S. Berry, J. Levinsohn, and A. Pakes. Automobile prices in market equilibrium. Econometrica, 63(4):841-890, 1995.

B. Caillaud and B. Julien. Competing in network industries: Divide and conquer. RAND J. Econ, 34:309-328, 2003.

J. E. Carranza. Product innovation and adoption in market equilibrium: The case of digital cameras. International Journal of Industrial Organization, 2010. ISSN 0167-7187.

Yong Chao and Timothy Derdenger. Mixed bundling in two-sided markets in the presence of installed base effects. Management Science, 2013. doi: 10.1287/mnsc.1120.1688. URL http://mansci.journal.informs.org/ content/early/2013/03/18/mnsc.1120.1688.abstract.

G. Crawford. The discriminatory incentives to bundle in the cable television industry. Quantitative Marketing and Economics, 6:41-78, 2008.

J. P. Dube, G. J. Hitsch, and P. K. Chintagunta. Tipping and concentration in markets with indirect network effects. Marketing Science, 29(2):216-249, 2010.

A. Elberse. Bye-Bye bundles: The unbundling of music in digital channels. Journal of Marketing, 74(3):107-123, 2010. 
B. R. Gordon. A dynamic model of consumer replacement cycles in the PC processor industry. Marketing Science, $28(5): 846-867,2009$.

G. Gowrisankaran and M. Rysman. Dynamics of consumer demand for new durable goods. Journal of Political Economy, 2013.

G. Gowrisankaran, M. Park, and M. Rysman. Measuring network effects in a dynamic environment. Technical report, NET Institute Working Paper No. 10, 2010.

A. Hagiu. Pricing and commitment by two-sided platforms. The RAND Journal of Economics, 37(3):720-737, 2006.

W. R Hartmann and H. S Nair. Retail competition and the dynamics of demand for tied goods. Marketing Science, $29(2): 366-386,2010$.

I. Hendel and A. Nevo. Measuring the implications of sales and consumer inventory behavior. Econometrica, 74 (6):1637-1673, 2006.

U. Kaiser and J. Wright. Price structure in two-sided markets: Evidence from the magazine industry. International Journal of Industrial Organization, 24(1):1-28, 2006.

M. L. Katz and C. Shapiro. Systems competition and network effects. Journal of Economic Perspectives, 8(2): 93-115, June 1994. doi: 10.1257/jep.8.2.93.

B. H Kobayashi. Does economics provide a reliable guide to regulating commodity bundling by firms? a survey of the economic literature. Journal of Competition Law and Economics, 1(4):707, 2005.

F. Mariuzzo, P. P. Walsh, and C. Whelan. Coverage of retail stores and discrete choice models of demand: Estimating price elasticities and welfare effects. International Journal of Industrial Organization, 28(5):555-578, September 2010.

R. P McAfee, J. McMillan, and M. D Whinston. Multiproduct monopoly, commodity bundling, and correlation of values. The Quarterly Journal of Economics, 104(2):371-383, 1989.

O. Melnikov. Demand for differentiated durable products: The case of the US computer printer market. Unpublished manuscript, Cornell University, 2001.

B. Nalebuff. Bundling as an entry barrier*. Quarterly Journal of Economics, 119(1):159-187, 2004.

A. Prasad, R. Venkatesh, and V. Mahajan. Optimal bundling of technological products with network externality. Management Science, 56(12):2224, 2010. 
J.C. Rochet and J. Tirole. Two-sided markets: a progress report. The RAND Journal of Economics, 37(3):645-667, 2006.

M. Rysman. Competition between networks: A study of the market for yellow pages. Review of Economic Studies, 71(2):483-512, 2004.

P. Schiraldi. Automobile replacement: a dynamic structural approach. The RAND Journal of Economics, 42(2): 266-291, 2011.

R. Schmalensee. Gaussian demand and commodity bundling. The Journal of Business, 57(1):211-230, 1984a.

R. Schmalensee. Gaussian demand and commodity bundling. The Journal of Business, 57(1):211-230, 1984b.

K.M. Sharpe and R. Staelin. Consumption effects of bundling: Consumer perceptions, firm actions, and public policy implications. Journal of Public Policy \& Marketing, 29(2):170-188, 2010.

B. Skrainka and K. Judd. High performance quadrature rules: How numerical integration affects a popular model of product differentiation. 2011.

R. Venkatesh, V. Mahajan, and V. Rao. The design and pricing of bundles: A review of normative guidelines and practical approaches. Handbook of Pricing Research in Marketing, Edward Elgar Publishing, Northampton, MA, pages $232-257,2009$.

S.B. Villas-Boas. Vertical relationships between manufacturers and retailers: Inference with limited data. The Review of Economic Studies, 74(2):625-652, 2007. 


\section{Appendix A: Model Fit and Robustness across Specifications}

Table 8 presents the seasonally adjusted raw sales data and the model predictions. As the table illustrates, our above model does quite nicely in predicting console and bundle sales. We determine the model has a prediction error of $0.44 \%$ for hardware and $6.59 \%$ for software, which indicates the model over predicts sales. We note that our prediction errors correspond quite favorably with other results Carranza [2010, Gowrisankaran et al. 2010] .

Table 8: Model Prediction Error

\begin{tabular}{llll}
\hline & Data & Model & Prediction Error \\
\hline Hardware Sales & $26,455,631$ & $26,540,532$ & $0.32 \%$ \\
Software Sales & $112,245,959$ & $115,579,373$ & $2.88 \%$ \\
Discounted Revenue & $2,112,371,134$ & $2,138,564,056$ & $1.2 \%$ \\
\hline
\end{tabular}

We also assess model fit by reporting the average hardware and software estimation error terms over the 45 month time period. Figure 12 presents this information. From this figure we see no evidence of systematic auto-correlation or heteroscedasticity of the average hardware or software error term over the time period.

Figure 12: Average Hardware $\left(\xi_{j t}\right)$ and Software $\left(\chi_{g t}\right)$ Structural Error by Month
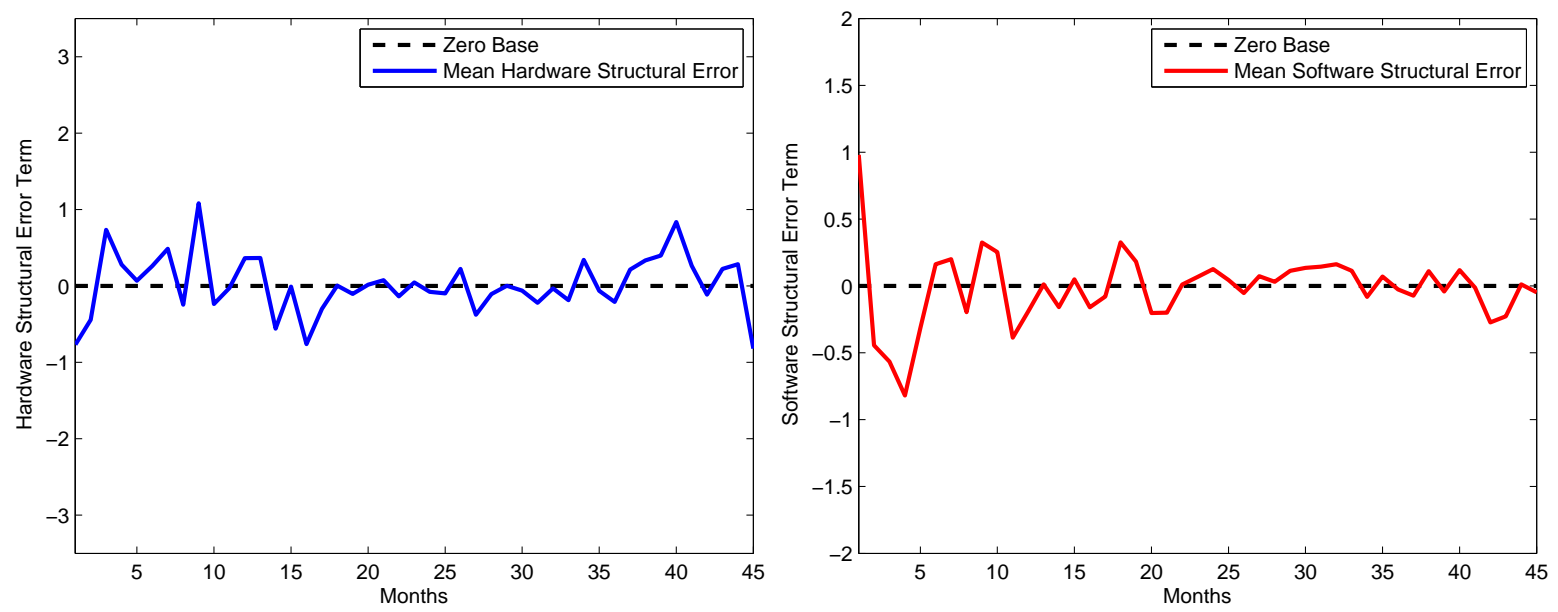

Next, we evaluate the robustness of the model to different specifications. We detail the results from four such specifications in Table 9 below. Note that each of the models below was a dynamic model with forward-looking consumers, and the estimation approach explicitly recovered consumer heterogeneity. We examined the effect of changes to our baseline model specification provided in the paper along the following dimensions:

- Bundle specific seasonal effects versus Hardware software seasonal effects: The rationale was to try and examine whether having separate seasonal effects for the bundle might produce different results than 
having the bundle seasonality accounted for by the sum of hardware and software seasonal effects.

- Dynamic Hardware: We evaluated the results from model specifications with the introduction of a separate set of forward-looking consumers in the hardware market. These consumers would enter during the holiday season (November and December) and not be present in the market during the other months.

- Myopic Software: Building on the baseline model, we investigated whether the presence of the myopic segment of consumers in the software market actually impacted the results qualitatively. We found that models with and without myopic consumers tended to produce similar results.

Overall, we find that across a wide range of model specifications, as detailed above, we find that the primary results of our model are robust, and that the conclusions we report in the main paper follow through across these different model specifications. 


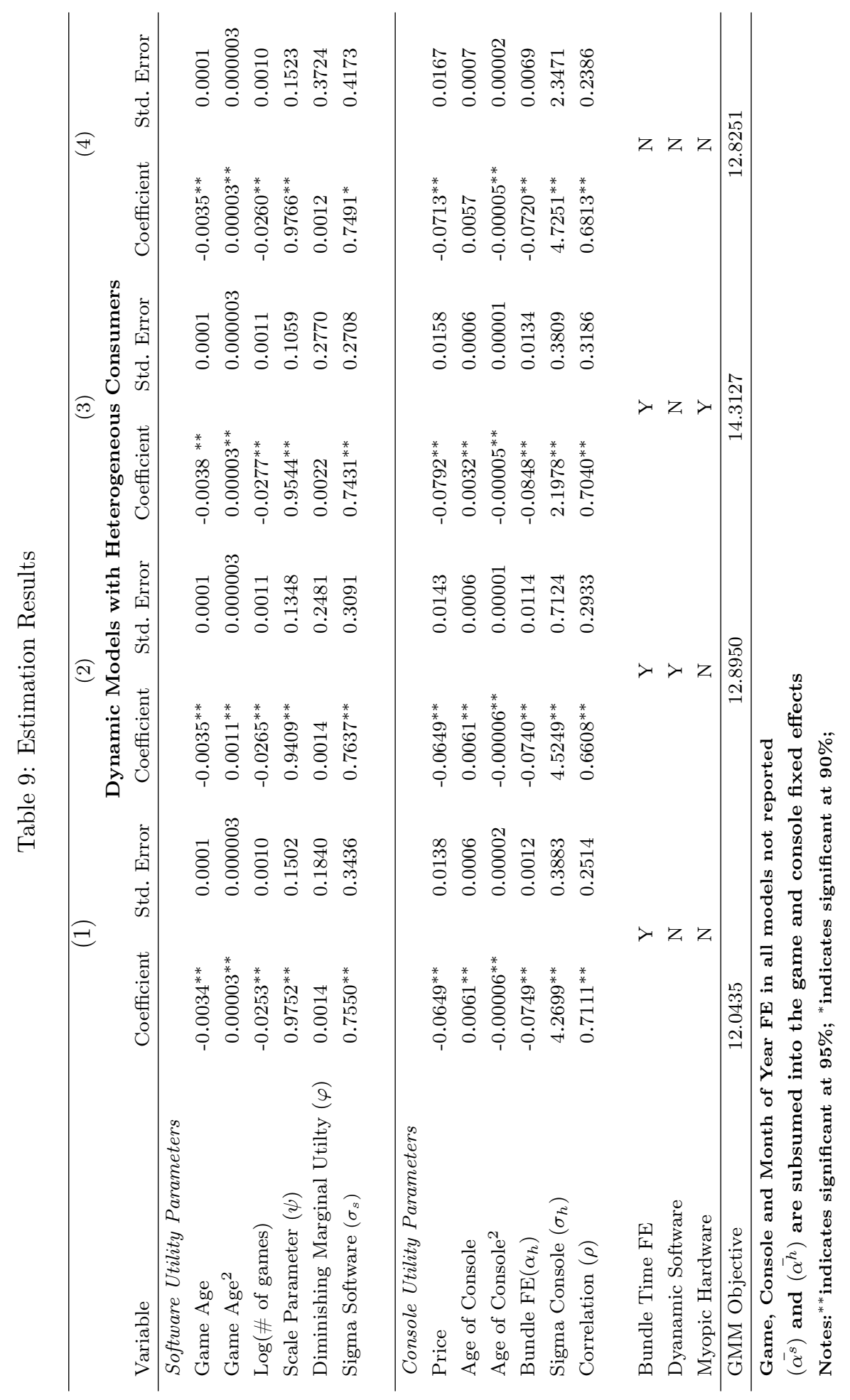




\section{Appendix B: Inclusive Value Specification}

The inclusive value is designed to capture the primary drivers of evolution of consumer utility in the software and hardware market. Our model assumes that a simple $\operatorname{AR}(1)$ process suffices to capture the major dynamic drivers of the inclusive value. To further determine the appropriateness of the IVS assumption, that consumers form expectations about next periods inclusive value with only information on today's inclusive value and an autoregressive specification, we plot the error term from the console and software decision problems $\left(\delta_{\bar{i}, t+1, c}-\right.$ $\left.\left(\gamma_{i, 1, c}+\gamma_{i, 2, c} \delta_{i, t, c}\right)\right)$ and $\left(\gamma_{\bar{i}, t+1, s}-\left(\gamma_{i, 1, s}+\gamma_{i, 2, s} \delta_{i, t, s}\right)\right)$. Recall that the IVS assumption tractably captures all of the information about future product introductions and prices as well as any key variables over which consumers have expectations; it is useful to note that despite such simplification, we find the error terms to be essentially unpredictable.

Figure 13: Hardware and Software Prediction Error
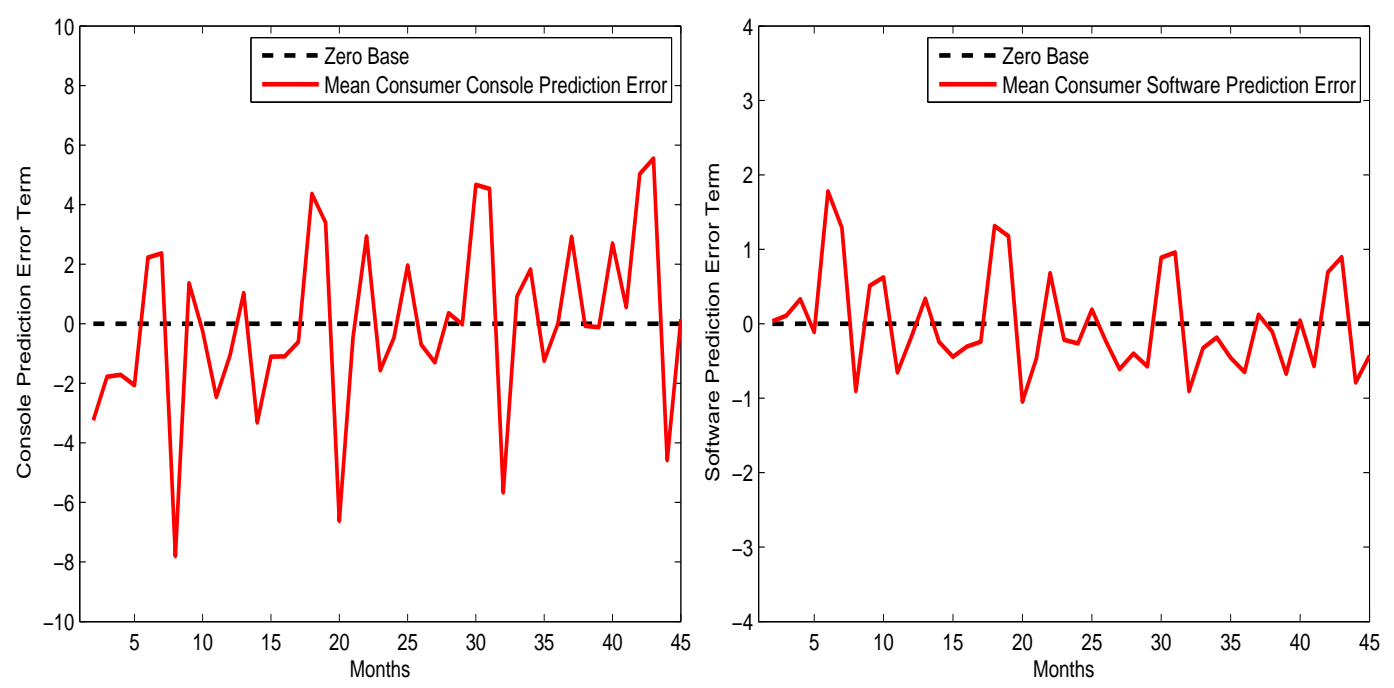

Figure 13 show errors terms that fluctuate quite drastically between negative and positive values as well as show no sign of any trend to becoming more negative or positive over time for both the console and software. These results inform us that a consumer's miscalculation of expected future values are driven by unanticipated changes in product attributes over time, leading to the conclusion that the IVS assumption we impose on consumer expectations about how the future evolves is reasonable. Moreover, it is useful to clarify what consumers are considering when making a hardware choice. In our current model, consumer on the hardware market make the buy-versus-wait tradeoff based on prices, entry of new console or bundles, expected utility from games (based both on availability and prices of games). Since all of the above factors are dynamically changing over time, we cannot accurately identify which one of them is the "primary" tradeoff. Especially, with heterogeneous consumers entering the market over time, the "primary" tradeoff itself may vary based on the period and other factors, e.g. consumers early in the console's life cycle may be waiting for more games, whereas late in the lifecycle, the primary inter- 
temporal tradeoff might come from price variations. However, our model is quite similar to GR in the sense that we collapse the future of the entire console market into an inclusive value that's heterogeneous across consumers. The model itself is general enough to allow both single purchases followed by exit, or repeat purchases where the consumers continues to stay in the market. The choice of which of these two is more appropriate depends on the details of the institutional setting. In our setting, corresponding to the present model, for the console market, we have prices of consoles, bundles, expectation of the utility from games (based on game availability, competition and price changes) all of which combine to constitute the "evolving characteristics." Furthermore, we do not expect that bundles constitute the primary option value, largely because sales from bundles is much lower than sales of pure consoles in virtually every time period, and there is significant variation in console prices, and especially in the software market. The changes in the software market are now folded back as a utility into our console utility specification, thus providing additional "smoothness." Finally, we illustrate below the seasonally adjusted plot of $\delta_{i t}$ for hardware over time. We seasonally adjust $\delta_{i t}$ by negating the estimated month fixed effects from hardware utility.

Figure 14: Changes in IVS $\left(\delta_{i t}^{h}\right)$ over time

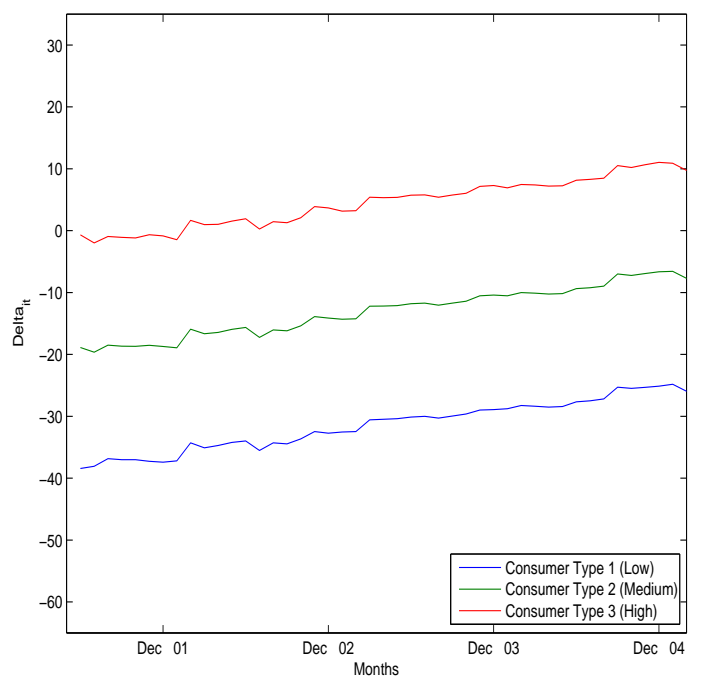




\section{Appendix C: Computational Details}

We use the following computational algorithm to begin the GMM procedure that provides a value to the GMM objective function, given a guess of the parameters: $\theta=\left(\alpha^{h}, \alpha^{s}, \alpha^{p, h}, \alpha^{p, s}, \alpha^{x, h}, \alpha^{w, s}, \Sigma\right)$. Note that we set $N_{\max }=$ 30.

\section{Overall GMM Objective Procedure:}

1. Obtain individual-specific parameters for each hardware and software segment of consumers, i.e. $\left(\alpha_{i}^{h}, \alpha_{i}^{s}\right)_{i=1}^{N_{s}}$ and using quadrature with $\left(\alpha^{h}, \alpha^{s}, \Sigma\right)$ as input. Make initial guess of $\lambda_{i, t}^{h}$.

2. Iterate through steps (1) - (15) in the software market procedure until tolerance is reached.

3. Update current estimate of indirect network effect in hardware market, $W_{i t}^{s}$, for each consumer and time period.

4. Iterate through steps (1) - (9) in the hardware market procedure with current estimate of $W_{i t}^{s}$ until tolerance is reached.

5. Update guess of $\lambda_{i, t}^{h}$.

6. Iterate through steps (2) - (5) until tolerance on $W_{i t}^{s}$ is reached

7. Compute the GMM objective function defined in equation (28) in the paper.

\section{Software Market Procedure}

1. Guess the mean flow utility of games, $v_{g t}^{s}$ for each $g \in \mathbf{S}_{\mathbf{t}}$, and for each period $t$. For consumer type $i$, obtain $v_{i g t}^{s}=v_{g t}^{s}+\alpha_{i}^{s}$.

2. The consumer segment mix is updated recursively for each consumer $i$ and period $t$ as follows:

$$
\lambda_{i,(t+1)}^{s}=\lambda_{i, 0}^{h}-\lambda_{i, t}^{h}
$$

3. Obtain the evolving inclusive value $\omega_{i t}^{s}$ for each type of consumer $i$ and period $t$, using the following equation:

$$
\omega_{i t}^{s}=\log \left(\sum_{g \in \mathbf{S}_{\mathbf{t}}} \exp \left(\frac{v_{i g t}^{s}}{1-\beta}+\alpha^{p, g} p_{g t}+\eta_{\tau(t)}^{s}\right)\right)
$$


4. Obtain the software evolving inclusive value coefficients through estimation of AR(1) regression:

$$
\omega_{i t+1}^{s}=\gamma_{i, 0}^{s}+\gamma_{i, 1}^{s} \omega_{i t}^{s}+\zeta_{i, t}^{s}
$$

5. Compute the utility of purchasing each game $g \in \mathbf{S}_{\mathbf{t}}$ in terms of the evolving inclusive value:

$$
\delta_{i g t}^{s}=\frac{v_{i g t}^{s}}{1-\beta}+\alpha^{p, g} p_{g t}+\eta_{\tau(t)}^{s}-\varphi\left(N_{g}+1\right)+\beta \mathbf{E}_{\omega_{\mathbf{i}}}\left[E V_{i}^{s}\left(\omega_{i, t+1}^{s}, N_{g}+1\right) \mid \omega_{i, t}^{s}\right]
$$

6. Compute the total software inclusive value, $\delta_{i t}^{s}$ based on period utility, flow utility and evolving inclusive value:

$$
\delta_{i t}^{s}\left(N_{g}\right)=\omega_{i t}^{s}-\varphi\left(N_{g}+1\right)+\beta \mathbf{E}_{\omega_{\mathbf{i}}}\left[E V_{i}^{s}\left(\omega_{i, t+1}^{s}, N_{g}+1\right) \mid \omega_{i, t}^{s}\right]
$$

7. Obtain consumer-specific expected value of not purchasing (and continuing) in the software market:

$$
\begin{aligned}
E V_{i}^{s}\left(\omega_{i t}^{s}, N_{g}\right)=\log \{\underbrace{\exp \left(\omega_{i t}^{s}-\varphi\left(N_{g}+1\right)+\beta \mathbf{E}_{\omega}\left[E V_{i}^{s}\left(\omega_{i, t+1}^{s}, N_{g}+1\right) \mid \omega_{i t}^{s}\right]\right)}_{\text {Purchase }} \\
+\underbrace{\exp \left(-\varphi\left(N_{g}\right)+\beta \mathbf{E}_{\omega}\left[E V_{i}^{s}\left(\omega_{i, t+1}^{s}, N_{g}\right) \mid \omega_{i t}^{s}\right]\right)}_{\text {No Purchase }}\}
\end{aligned}
$$

where the expectation is taken based on the coefficients recovered through the AR(1) regression above. The state space is defined as: $N_{g} \in\left\{1,2, \ldots, N_{\max }\right\}$. Note that when the consumer already holds $N_{g} \geq N_{\max }$ videogames, then the state continues to be $N_{\max }$ and is not incremented with a purchase. Thus, the state transition can more accurately be represented as: $N_{g}^{(t+1)}=\min \left\{N_{g}^{t}+\left(\sum_{g \in S_{t}} d_{g t}\right), N_{\max }\right\}$.

8. Calculate probability of consumer $i$ purchasing in period $t$ :

$$
P_{i t}^{s}\left(\delta_{i t}^{s}, N_{g}\right)=\frac{\exp \left(\delta_{i t}^{s}\left(N_{g}\right)\right)}{\exp \left(E V_{i}^{s}\left(\delta_{i t}^{s}, N_{g}\right)\right)}
$$

9. Calculate the probability of consumer $i$ purchasing product $k$ in period $t$ conditional on making a purchase as:

$$
P_{i k t}^{s}=\frac{\exp \left(\delta_{i g t}^{s}\right)}{\exp \left(\delta_{i t}^{s}\right)}
$$

10. Determine $\Lambda_{i t}\left(N_{g}\right)$, the fraction of consumers of type $i$ holding $N_{g}$ number of games in period $t$, where $N_{g} \in\left\{1, \ldots, N_{\max }\right\}$.

11. Compute the forward looking consumer $i$ 's purchase probability share for each product $k \in \mathbf{S}_{\mathbf{t}}$ in each period 
$t$ as:

$$
s_{i k t}^{\text {dynamic }}=P_{i k t}^{s}\left(\sum_{N_{g}=0}^{N_{\max }} P_{i t}^{s}\left(\delta_{i t}^{s}, N_{g}\right) \Lambda_{i t}\left(N_{g}\right)\right) .
$$

12. If the model has myopic consumers, calculate the myopic consumer $i$ 's purchase probability for each product $k$ in period $t$ as:

$$
s_{i k t}^{\text {myopic }}=\frac{\exp \left(v_{i k t}^{s}+\alpha^{p, g} p_{s, t}+\eta_{\tau(t)}^{s}\right)}{1+\left(\sum_{k \in \mathbf{S}_{\mathbf{t}}} \exp \left(v_{i k t}^{s}+\alpha^{p, g} p_{s, t}+\eta_{\tau(t)}^{s}\right)\right)} .
$$

13. The overall market share for product $k$ in period $t$ is determined by integrating out the individual-specific coefficients, or in a quadrature approach, by weighting appropriately across consumer types (reported results are from the quadrature approach):

$$
\hat{s}_{k t}=\left[1-\mathbf{I}\left(\text { seasonal }_{t}\right)\right] \sum_{i \in \mathbf{I}_{\mathbf{t}}} \lambda_{i, t}^{s} s_{i k t}^{\text {dynamic }}+\mathbf{I}\left(\text { seasonal }_{t}\right)\left[\frac{2}{3} \sum_{i \in \mathbf{I}_{\mathbf{t}}} \lambda_{i, t}^{s} s_{i k t}^{\text {dynamic }}+\frac{1}{3} \sum_{i \in \mathbf{I}_{\mathbf{t}}} \lambda_{i, t}^{s} s_{i k t}^{\text {myopic }}\right]
$$

14. Update the flow utilities corresponding to a product-period combination similar to BLP, based on how close the model is to observed data:

$$
v_{k t}^{s}=v_{k t}^{s}+\log \left(\frac{s_{k t}^{s, D A T A}}{\hat{s}_{k t}^{s}}\right)
$$

15. Iterate through Steps (3)-(13) until tolerance is reached.

16. Recover the indirect network effect for the hardware market, $W_{i t}^{c}=E V_{i}^{s}\left(\delta_{i t}^{s}, N_{g}=0\right)$ and $W_{i t}^{b}=E V_{i}^{s}\left(\delta_{i t}^{s}, N_{g}=1\right)$

\section{Hardware Market Procedure}

1. Guess $F_{k t}^{h}$ for each $k \in \mathbf{J}_{\mathbf{t}} \cup \mathbf{B}_{\mathbf{t}}$, and for each period $t$.

2. Obtain $\delta_{i k t}^{h}$ for each type of consumer $i$ for each hardware product $k$ and period $t$, using the following equation:

$$
\delta_{i k t}^{h}=\left\{\begin{array}{c}
\frac{F_{k t}^{h}+\alpha_{i}^{h}}{(1-\beta)}+\alpha^{p, h} p_{k, t}+\eta_{\tau(t),}^{h} \quad k \in \mathbf{J}_{\mathbf{t}} \\
\frac{F_{k t}^{h}+\alpha_{i}^{h}+\alpha_{i}^{s}+\alpha^{b}}{(1-\beta)}+\alpha^{p, h} p_{k, t}+\eta_{\tau(t),}^{b}, \quad k \in \mathbf{B}_{\mathbf{t}}
\end{array}\right.
$$

3. Compute the inclusive value for each consumer:

$$
\delta_{i t}^{h}=\log \left(\sum_{k \in \mathbf{J}_{\mathbf{t}} \cup \mathbf{B}_{\mathbf{t}}} \exp \left(\delta_{i k t}^{h}\right)\right)
$$


4. Obtain the hardware coefficients through estimation of AR(1) regression:

$$
\delta_{i, t+1}^{h}=\gamma_{i, 0}^{h}+\gamma_{i, 1}^{h} \delta_{i, t}^{h}+\zeta_{i, t}^{h}
$$

5. Obtain consumer-specific expected value of not purchasing (and hence continuing) in the hardware market:

$$
E V_{i}^{h}(\delta)=\log \left(\exp (\delta)+\exp \left(\beta \mathbf{E}\left[E V_{i}\left(\delta^{\prime}\right) \mid \delta\right]\right)\right)
$$

where the expectation is taken based on the coefficients recovered through the $\mathrm{AR}(1)$ regression above.

6. The model-predicted purchase probability share for each product $k$ in each period $t$ is then given for consumer $i$ to be:

$$
\hat{s}_{i k t}^{h}=\frac{\exp \left(\delta_{i t}^{h}\right)}{\left[\exp \left(E V_{i}^{h}\left(\delta_{i t}^{h}\right)-\kappa\right)\right]} \frac{\exp \left(\delta_{i k t}^{h}\right)}{\exp \left(\delta_{i t}^{h}\right)}
$$

7. The overall market share for product $k$ in period $t$ is determined by integrating out the individual-specific coefficients, or in a quadrature approach, by weighting appropriately across consumer types:

$$
\hat{s}_{k t}^{h}=\sum_{i=1}^{N_{s}} \lambda_{i, t}^{h} s_{i k t}^{h}
$$

8. The consumer segment mix is updated according to the equation, recursively for each consumer $i$ and period $t:$

$$
\lambda_{i,(t+1)}=\lambda_{i, t}\left(1-\sum_{k \in \mathbf{J}_{\mathbf{t}} \cup \mathbf{B}_{\mathbf{t}}} \hat{s}_{i k t}^{h}\right)
$$

9. The flow utilities corresponding to a product-period combination are then updated similar to BLP, based on how close the model is to the observed data:

$$
F_{k t}^{h}=F_{k t}^{h}+\log \left(\frac{s_{k t}^{h, D A T A}}{\hat{s}_{k t}^{h}}\right)
$$

10. Iterate through Steps (2)-(9) until tolerance is reached. 